\title{
Theoretical Analysis for Variable Delivery Flow External Gear Machines Based on Asymmetric Gears
}

\author{
Ram Sudarsan Devendran, Andrea Vacca, \\ Maha Fluid Power Research Center, School of Mechanical Engineering, Purdue University \\ 1500 Kepner Dr, Lafayette, IN 47905, USA \\ E-mail: ramsudarsand@gmail.com, avacca@purdue.edu
}

\begin{abstract}
This paper presents an analytical approach for the analysis of a new working concept for variable delivery external gear machines. The proposed design principle encompasses all the advantages of conventional fixed displacement external gear machines, along with a simple mechanism to change the displaced flow per shaft revolution. The variation in the displaced flow is achieved by realizing a variable timing for the connections of each tooth space volume (volume between two adjacent teeth of the gears) with the inlet and outlet. This is achieved through a movable slider placed at the gear lateral sides. The position of the slider determines the amount of flow displaced by the unit per revolution. Analytical expressions which define the range of percentage variation in flow displacement that can be achieved based on a generic asymmetric design of the gears are presented. The paper presents a detailed analytical derivation for geometric displacement, flow delivered at the outlet and shaft torque required during operation as well as flow nonuniformity/pulsation. The analytical expressions were compared with proof of concept test. Experimental results were in line with the analytical prediction and show a 32\% variation in displacement. These positive results show promising potentials for the working of a new concept for variable delivery external gear machine.
\end{abstract}

\section{Keywords}

External gear pumps; variable displacement; variable delivery; geometric displacement, input shaft torque, energy efficiency; volumetric efficiency

\begin{tabular}{lll}
\multicolumn{2}{l}{ Nomenclature } & Units \\
Symbol & Description & $\mathrm{Nm}$ \\
$\mathrm{M}$ & Torque acting on gear & $\mathrm{I} / \mathrm{min}$ \\
$\mathrm{Q}$ & Flow rate at the outlet of the machine & $\mathrm{cm}^{3}$ \\
$\mathrm{~V}$ & Geometric displacement of the machine & $\mathrm{W}$ \\
$\mathrm{W}$ & Work done to displace $d v$ over a pressure barrier $\Delta p$ & $\mathrm{~mm}$ \\
$\mathrm{~b}$ & Face width of the gears & $\mathrm{mm}$ \\
$l_{c}$ & Length of the working part of the coast side line of action & $\mathrm{mm}$ \\
$l_{d}$ & Length of the working part of the drive side line of action & $\mathrm{bar}$ \\
$p$ & Pressure & $\mathrm{mm}$ \\
$r_{a}$ & Addendum radius & $\mathrm{mm}$ \\
$r_{0}$ & Pitch radius & $\mathrm{mm}$ \\
$r_{b}$ & Base circle radius & $\mathrm{mm}$ \\
$r_{r}$ & Root circle radius & $\mathrm{mm}$ \\
$r_{b d}$ & Drive side base circle radius & $\mathrm{mm}$ \\
$r_{b c}$ & Coast side base circle radius & $\circ$ \\
& Pressure angle & $\circ$
\end{tabular}

(C) 2016. This manuscript version is made available under the Elsevier user license http://www.elsevier.com/open-access/userlicense/1.0/ 


\begin{tabular}{|c|c|}
\hline$\alpha_{0 d}$ & Drive pressure angle \\
\hline$\alpha_{0 c}$ & Coast pressure angle \\
\hline$\beta$ & Level of displacement \\
\hline$\Delta p$ & Pressure difference \\
\hline$\delta_{Q}$ & Non-uniformity in flow rate \\
\hline$\delta_{M}$ & Non-uniformity in input torque \\
\hline$\theta_{D}$ & $\begin{array}{l}\text { Angular position at which the TSV begins to be trapped between the points } \\
\text { of contact of the teeth. }\end{array}$ \\
\hline$\theta_{S}$ & $\begin{array}{l}\text { Angular position at which the TSV ends to be trapped between the points of } \\
\text { contact of the teeth. }\end{array}$ \\
\hline$\eta_{v}$ & Volumetric efficiency \\
\hline
\end{tabular}

$\begin{array}{ll}\text { Abbreviations } \\ \text { Symbol } & \text { Description } \\ \text { EGM } & \text { External Gear Machine } \\ \text { FD } & \text { Fixed displacement } \\ \text { HP } & \text { High Pressure } \\ \text { LP } & \text { Low Pressure } \\ \text { TR } & \text { Trapped Volume } \\ \text { TSV } & \text { Tooth Space Volume } \\ \text { VD } & \text { Variable Displacement } \\ \text { avg } & \text { Average } \\ \text { max } & \text { Maximum value } \\ \text { min } & \text { Minimum value }\end{array}$

\section{Introduction}

Numerous fluid power applications, fuel injection and fluid transport systems employ External Gear Machines (EGMs) for their distinguished advantages such as ease of manufacturability, compactness, reliability, efficiency and low cost. The working of the EGM is very simple as can be explained using the schematic of Figure 1, typical for high pressure EGM ( $p>$ $100 \mathrm{bar}$ ). As the shaft rotates, the fluid is transferred from the inlet to the outlet through the periphery of the gears, and the displacement of the fluid is achieved by the meshing of the teeth. At gears lateral side, grooves are machined on the pump case or on the lateral bushing in case of pressure compensated pumps as shown in Figure $1(B)$. These grooves realize proper connections of the inter-teeth volumes trapped between points of contact with the inlet/outlet ports, thus avoiding these volumes to be isolated from the inlet/outlet environment during the meshing process. As a consequence, the grooves play an instrumental role in minimizing the internal pressure overshoots and localized cavitation effects shown in Figure $1(C)$, without compromising the volumetric capacity during operation. Clearly, the principle of the operation of EGMs restricts to delivering only a fixed amount of fluid per unit revolution (fixed displacement unit). The inherent nature of EGMs being fixed displacement units makes this machine design inapplicable to fluid power systems with energy efficient architecture applications ([1], [2]). Particularly, these systems employ variable displacement (VD) units (capable of providing flow on demand by being able to change the amount of fluid delivered per unit revolution) which have proven to be offering significant energy savings as opposed to systems with fixed displacement units. Even though EGMs are traditionally fixed displacement type, they are significantly less expensive than their VD counterparts and hence have been widely and successfully used in several hydraulic applications till date. 


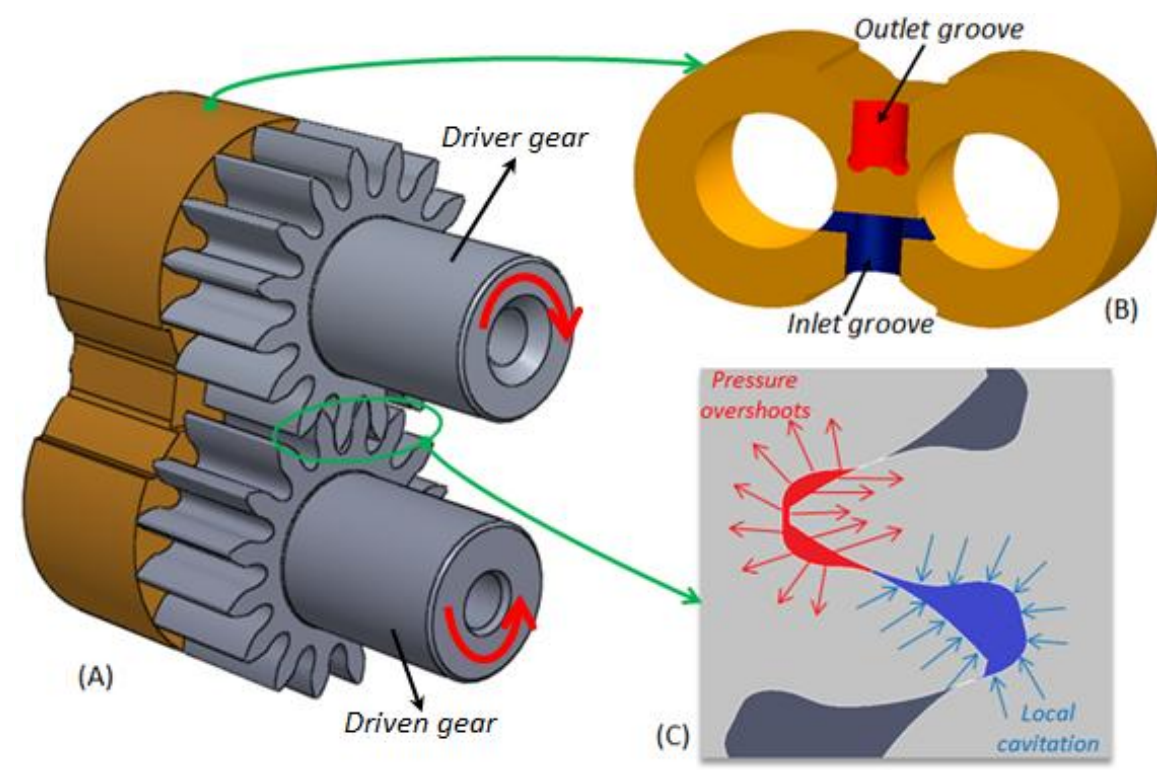

Figure 1: Gear set (A) Gears meshing with each other; (B) Detail of the grooves in the lateral bushings; (C) Detail of the meshing process showing the regions of pressure peaks and local cavitation.

In an effort to obtain an innovative, working and inexpensive VD EGMs, the authors have presented a very simple concept (as briefly explained in section 2 and provided in detail in [3]) which could bring about significant energy savings (about 3540\%). In [3], the authors proposed the idea, with a qualitative explanation of the delivery flow, input torque etc. on the basis of simulations and preliminary proof of concept tests. After this preliminary work, the authors implemented a working prototype equipped with both a manual system for adjusting the flow and an absolute pressure limiter [4]. In the current paper, an analytical approach for predicting the performance of VD-EGMs is provided for the very first time.

Literature review pertaining to analytical studies of EGMs shows that there are only few works which explain their performance in great detail. (Beacham, 1946) presented, a simplified graphical approach to understand the effect of grooves in the lateral bushing on the flow ripple at the delivery is provided. This study also discusses the impact of casing wear on the volumetric performance of the EGM. (Bonacini, 1961) derived an analytical expression for the kinematic flow ripple of EGM, this formulation was subsequently extended for the derivation of the input torque by (Ivantysyn et al, 2003). Using a similar approach, (Manring et al, 2005) analyzed the performance of the kinematic (or theoretical) flow ripple with different number of teeth on the driver and driven gears. The study concludes that the number of teeth on the driver gears dictates the flow ripple at the delivery and hence a higher number of teeth on the driver gear are recommended to reduce flow pulsations. (Negrini, 1996) and (Schwuchow, 2002) presented an EGM design with involute gears and zero backlash in order to reduce fluid borne noise emissions. In recent years, the research on EGM as presented by (Morselli, 2008) and also adopted by popular manufacturers like Marzocchi ("Elika"), Settima ("Continuum"), Bosch-Rexroth ("Silence Plus") [12] etc. has been predominantly towards reducing noise emissions by the implementation of helical gears with cycloidal profile as opposed to conventional involute profiles. However, the design of EGMs with helical gears still posts some limits, such as the limited volumetric efficiency as well as a more delicate axial balance mechanism for the compensation of the internal lateral gaps (Morselli, 2005). More recently, the design of the gears represented by (Morselli, 2015), which follow a highly asymmetrical design of involute teeth with a small helix angle has been adopted by Settima and Turolla ("Shhark") to tackle the problem of noise emissions with an approach alternative to EGMs with helical rotors. However, none of the previously mentioned works have made an effort to implement variable displacement/delivery flow in EGMs taking advantages of the features of the tooth profile in EGMs.

There have been only a very few patents that fall under the broader classification of design of variable displacement EGMs as described in this paragraph. Particularly, patents $[15,16]$ dealt with moving the gears in the radial direction to achieve a variation in displacement. These patents however do not describe the mechanism by which they would seal the displacement chambers while moving the gears axis of rotation. Hence, these solutions would lead to poor volumetric efficiency at high operating pressure. On the other hand, the designs described in the patents [17 - 20] achieve variable displacement by moving the gears along their axis (staggered arrangement of the gears) thereby achieving partial meshing of the gears. Commercial solutions are available in automotive transmission systems [21], thus for low pressure 
applications. For higher operating pressure, these designs become complicated with numerous additional parts required to achieve the partial engagement of the gears and would result in an expensive unit that is difficult to manufacture compared to existing gear machines. The volumetric performance of these designs is also questionable due to the lack of proper sealing of the displacement chambers.

In the present research, the authors present a solution for implementing a variable displacement in EGMs based a variable timing of the connections of the displacement chambers (the tooth space volumes, TSVs) with the inlet/output ports. Firstly presented in [3], the proposed concept for VD-EGMs maintains all the advantages of traditional EGMS, but it also offers a range of variation of the delivered flow. In this paper, a novel effort has been made to analytically describe the operation of the proposed principle of achieving variable timing in EGMs. Firstly, analytical expressions for the locations of the contact points of the gears have been derived based on the geometry of the gears are presented in section 3 . The locations of these points prove to be of utmost importance, as they define the extent to which the displacement can be reduced. Secondly, analytical derivations for geometric displacement, flow delivered at the outlet and shaft torque required during operation has been provided both for max and min displacement (section 4). The expressions pertaining to the nonuniformity/pulsations in flow rate and hence the shaft torque has also been presented for max and min displacement in section 5. The analytical expressions were validated with the optimal design results presented in [3] and briefly shown in section 6. A detailed analysis of the forces and torque explaining the reduction in energy consumption obtained due to variable displacement is presented in section 7. Experimental validation supporting the analytical finding of higher flow pulsations at min displacement has been presented in section 8.

\section{Concept for Achieving Variable Displacement in EGMs}

The novel concept for achieving variable delivery in EGMs is based on achieving variable timing for the connections of the TSVs with the inlet/outlet grooves. The "slider" - a movable element which can be placed in the pump case or in the bearing block (for pressure compensated designs), as shown in Figure 2(A), is capable of moving in the direction as depicted in Figure 2(A) and hence realizes a variation in the timing of the connections. The position of the slider determines the amount of flow displaced by the unit per revolution, for both the cases of pumps and motors.

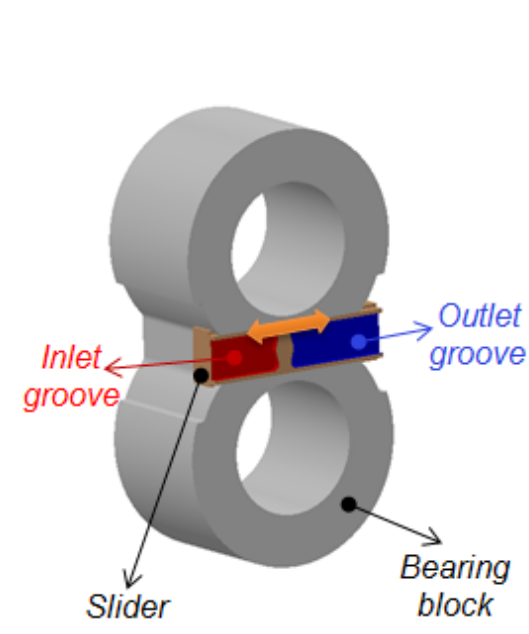

(A)

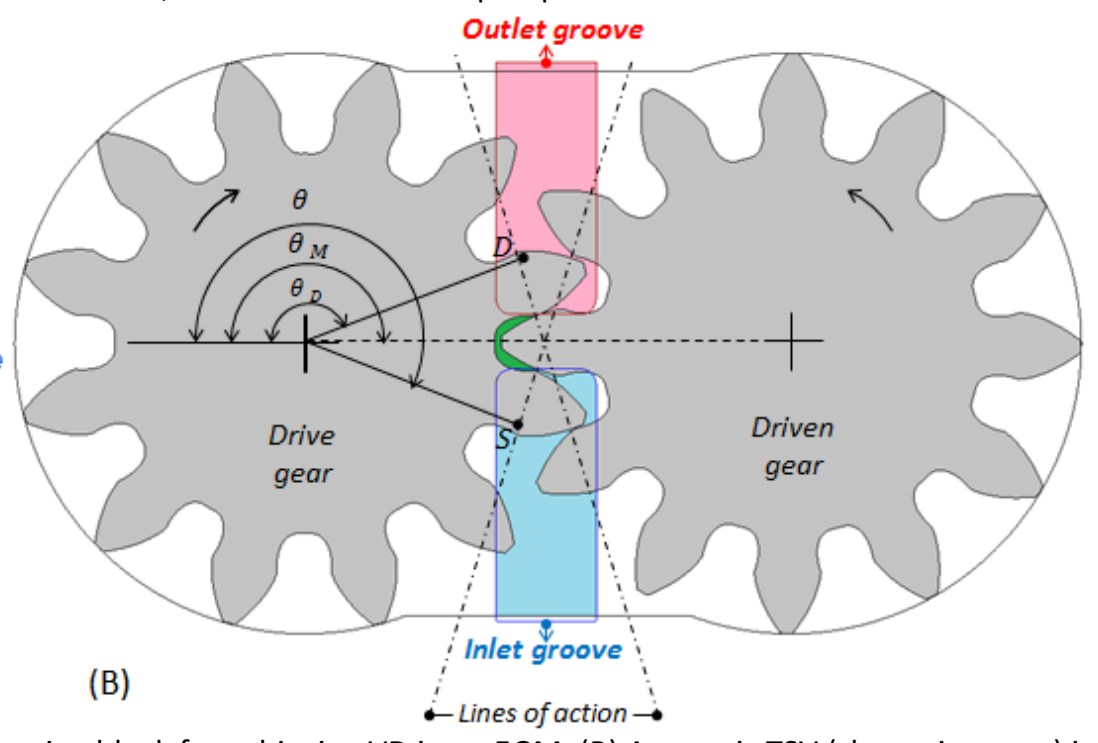

Figure 2: (A) Slider placed inside the bearing block for achieving VD in an EGM. (B) A generic TSV (shown in green) in the meshing zone and the grooves positioned for max displacement.

In order to understand the working of the proposed concept for VD-EGMs, the displacing action of a typical EGM is depicted in Figure 3. The meshing region of the gears can be identified as the angular region represented by $\theta$ in Figure 3 . Within $\theta$, there exists points $D$ and $S$ in the line of action of the gears, where the TSV is trapped between the points of contact. The displacing action is realized by means of inlet and outlet grooves (a simplified representation is shown in Figure 2(B)). In standard EGMs, to achieve maximum volumetric capacity, the commutation of TSVs between inlet and outlet groove is realized when the volume is minimum (Point $\mathrm{M}$, Figure 3). Therefore, the max volume that can be displaced can be qualitatively shown as the volume, $\left(V_{\max }-V_{M}\right)$ as shown in Figure 3. 


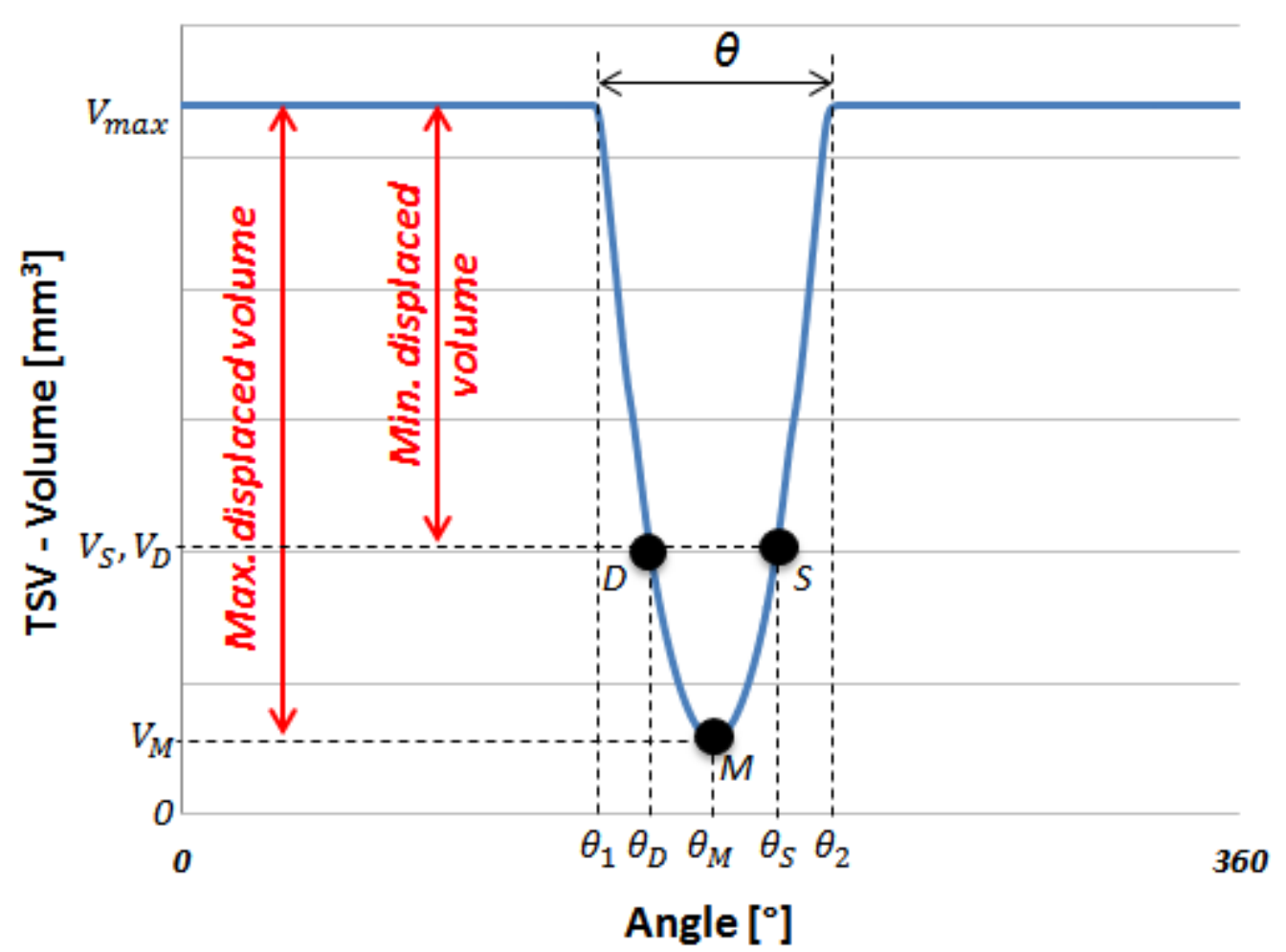

Figure 3: The progression of the TSV as a function of shaft rotational angle.

The movement of the slider of Figure 2(A) permits the variation in the position of the grooves and hence affects the angular position at which the commutation between the connections of each TSV with the inlet/outlet ports occurs.

Using Figure 3 and Figure 4, the effect of different positions of the slider can be explained. For achieving maximum displacement, the TSV is connected to the delivery for the angular interval $\left(\theta_{1}-\theta_{M}\right)$ and to the suction for the angular interval $\left(\theta_{M}-\theta_{2}\right)$. For conventional gears with symmetric profile $\theta_{M}$ falls at the midpoint of $\theta_{1}$ and $\theta_{2}$, hence the TSV is connected to the inlet and outlet for equal intervals of time, therefore, in this condition the unit displaces the maximum amount of flow. However, to achieve reduced flow delivery, the slider can be positioned closer to the inlet port. In this configuration the TSV remains connected to the outlet port for a longer angular interval, $\left(\theta_{1}-\theta_{S}\right)$ and to the suction for a smaller angular interval, $\left(\theta_{S}-\theta_{2}\right)$. Each TSV remains connected to the outlet port for a first portion of the filling process, (after it reaches the minimum volume point, angular interval $\left(\theta_{M}-\theta_{S}\right)$ ), wherein a part of the fluid already delivered to the outlet is taken back into the TSV which acts as additional dead volume. Due to this additional dead volume, each TSV is capable of displacing a reduced volume of fluid. Graphically, the additional dead volume can be represented by $\left(V_{S}-V_{M}\right)$. Therefore the effective fluid displaced to the outlet at reduced displacement can be represented by the volume, $\left(V_{\max }-\right.$ $\left.V_{S}\right)$. 


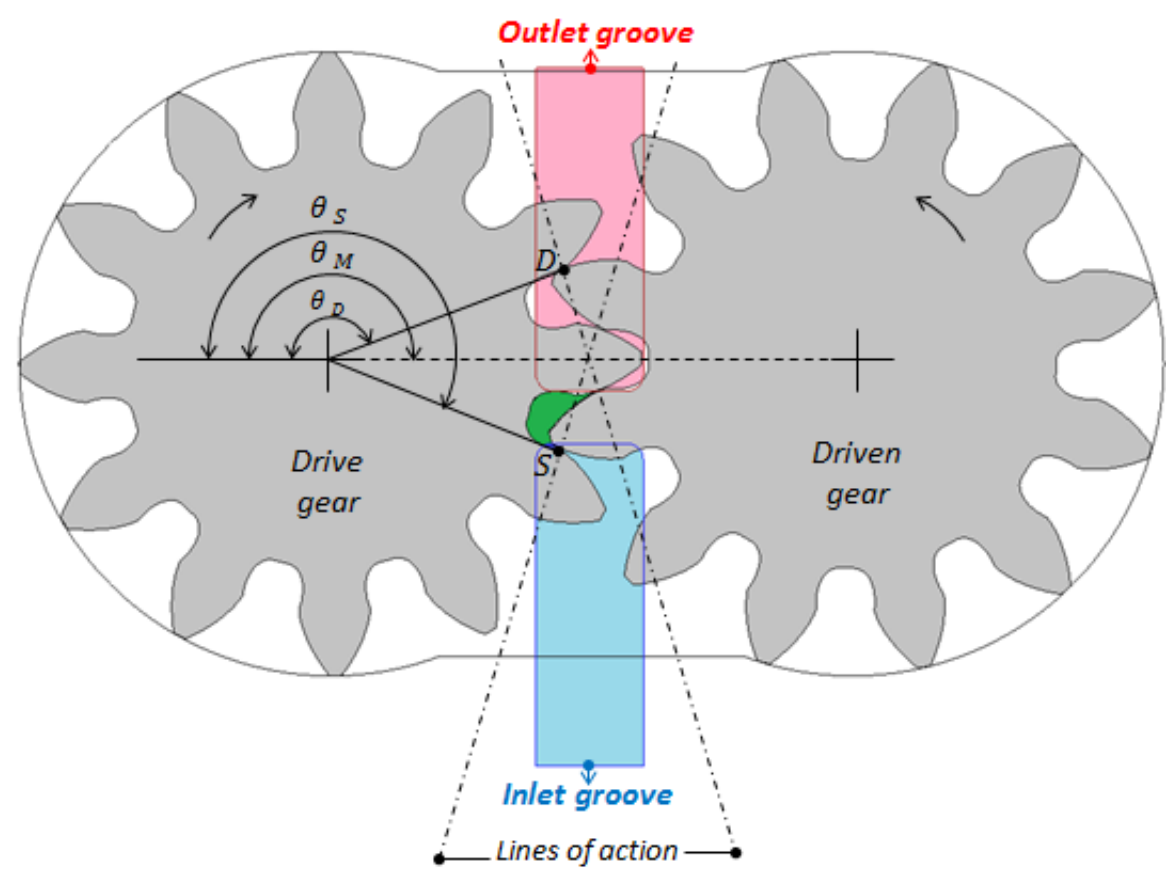

Figure 4: Slider position moved towards the suction (commutation at point S) enabling delivery of reduced displacement.

Conceptually, a reduced displacement can be achieved also by moving the slider to the outlet port and ensuring the commutation of the TSV from the inlet to the outlet occurs at Point D. However, with this alternative mode of varying displacement additional undesired effects due to fluid aeration that may occur during the process.

In essence, the proposed design implements on the EGM architecture the principle of "virtually variable displacement machine" (also referred often as "digital pump") as applied in piston machines [22, 23]. In piston machines, this concept is based on the usage of very fast ON/OFF valves to control the timing of the opening/closing of the inlet/outlet valves of each piston (displacement) chamber. Particularly, both the inlet and outlet valve can be opened and closed at intermediate points of the movement of the piston, different from the top/bottom end points. In this way, the volume of fluid actually displaced by the chamber can be varied. The concept is quite promising for hydrostatic machines also because the additional compressibility losses associated with higher dead volumes are minimal thanks to the high bulk modulus of the working fluids. On the contrary to virtually variable displacement, the proposed EGM design does not require fast on/off valves to determine the timing of the connections of each displacement chamber. In fact, the position of the "slider" can be directly used to control the timing and hence the displaced flow: the position of the slider determines the displacement, and there is no need to vary it during the displacement cycle of each displacement chamber. The main innovative feature of the design is supported by the fact that the displacement chambers are connected to the high pressure region for longer period of time during the meshing process: directly translates to significant torque advantage to the gear shafts and hence would result in reduction in input shaft torque requirement (less energy consumed). Since the proposed concept does not involve an addition of large number of parts to achieve variable displacement, the design of the VD-EGM would be very inexpensive as compared to that of other VD-units (typically almost 10 times more expensive) available in the market. A more extensive description of the proposed concept for VD-EGM and its advantages are provided in [3].

From Figure 3, one can easily notice that the maximum flow variation range greatly depends on the distance of the points $D$ and $S$ from the point $M$ on the curve representative of the TSV variation. Qualitatively, the larger the distance of the points $\mathrm{D}$ and $\mathrm{S}$ from point $\mathrm{M}$, the larger is the reduction in displacement that can be achieved. For this purpose, as described in [3] gears with asymmetric profile operating in dual flank contact (Figure 5) was considered, to maximize the range of flow variation.

Even though the proposed design concept for variable displacement EGMs offers lower flow rates at the expense of lower input shaft torque at reduced displacement [3], there are some drawbacks intrinsic to the design. Particularly, it can be easily noted that the proposed concept has a limitation on the range of flow variation that can be achieved. The range of flow variation as previously explained depends on the location of the Points $D$ and $S$ that represent the extreme points on the line of contact. The proposed design as explained in the later section of the paper is able to achieve a min displacement 
to $68 \%$. If the displacement has to be reduced further then, the points $\mathrm{D}$ and $\mathrm{S}$ should move farther from Point $\mathrm{S}$, which would result in gears with larger number of thinner and weaker teeth [3]. The achievable partial displacement variation is still attractive to several hydraulic applications such as charge pumps, fan drive units etc. Another drawback to the design is posed by the additional leakage through the interface between the outlet and inlet through the gap between the slider (surface of the slider not facing the gears) and the bearing block (surface of the slider not facing the gears) [3, 4, 24]. A larger delivery flow oscillation as well as input shaft torque pulsation would be observed at reduced displacement as described in Section 5 of this paper.

(A)

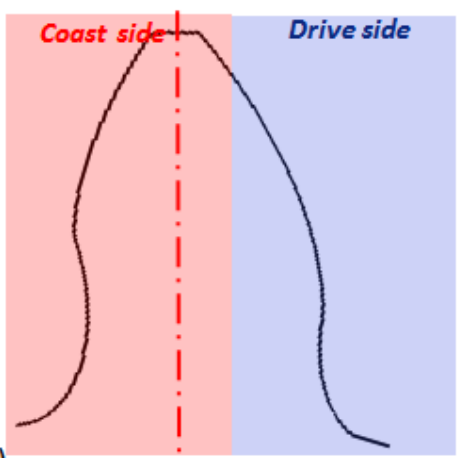

(B)

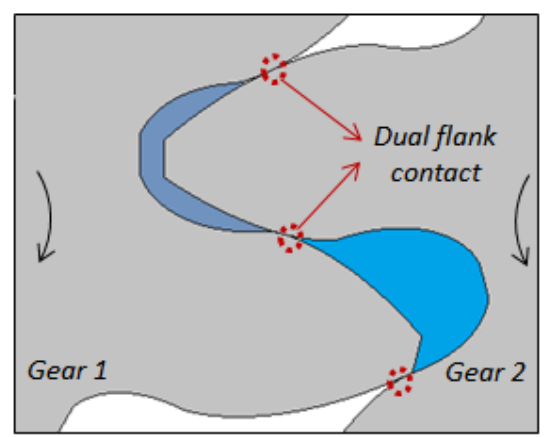

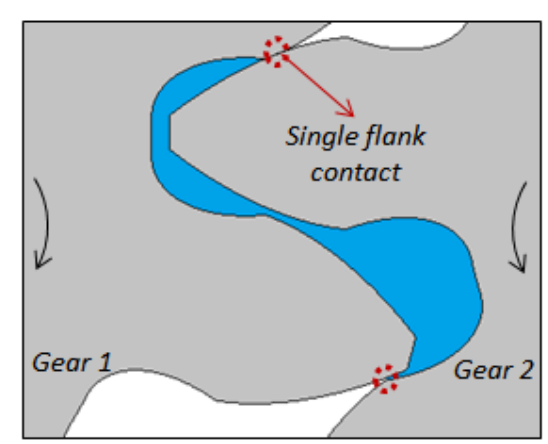

(C)

Figure 5: (A) Asymmetric gears depicting the drive and coast side of the tooth; (B) Asymmetric gears with dual flank contact;

(C) Symmetric gears with single flank contact .

\section{Analytical Determination of the Points of Contact}

The points of contact which define the angular region within which the TSV is trapped can be determined using the geometry of the involute curves and the line of action. In this section a generalized approach for identifying the points of contact for an asymmetric teeth profile is considered.

The asymmetric teeth profile is defined on the basis of two different pressure angles which control the shape of the tooth. A detailed description of the generation of the asymmetric tooth is provided in, $([3],[25,26])$, but this section focuses on the calculation of the points of contact based on trigonometric functions and gear geometry. Each tooth can be differentiated as drive side and coast side based on two different pressure angles, with the drive side pressure angle $\left(\alpha_{0 d}\right)$ always considered to be larger than the coast side $\left(\alpha_{0 c}\right)$, since power is transmitted from the drive gear to the driven gear via the drive side of the tooth. With reference to Figure 6, the addendum/outside circle of the gears is represented in green, the pitch circle in black and the base circles (drive side and coast side) are represented in blue. The drive side base circle radius can be expressed as,

$r_{b d}=r_{0} \cdot \cos \alpha_{0 d}$

Similarly, the coast side base circle radius can be expressed as,

$r_{b c}=r_{0} \cdot \cos \alpha_{0 c}$.

Since the gears are assumed to operate in dual flank contact configuration as shown in Figure 5(A), there are two lines of action corresponding to the drive and coast pressure angles (also depicted in Figure 6). The drive side line of action is represented by the red line $K_{1} L_{1}$, formed by the common tangent between the drive side base circles. Similarly, the coast side line of action is represented by the red line $K_{2} L_{2}$ formed by the common tangent between the coast side base circles. 


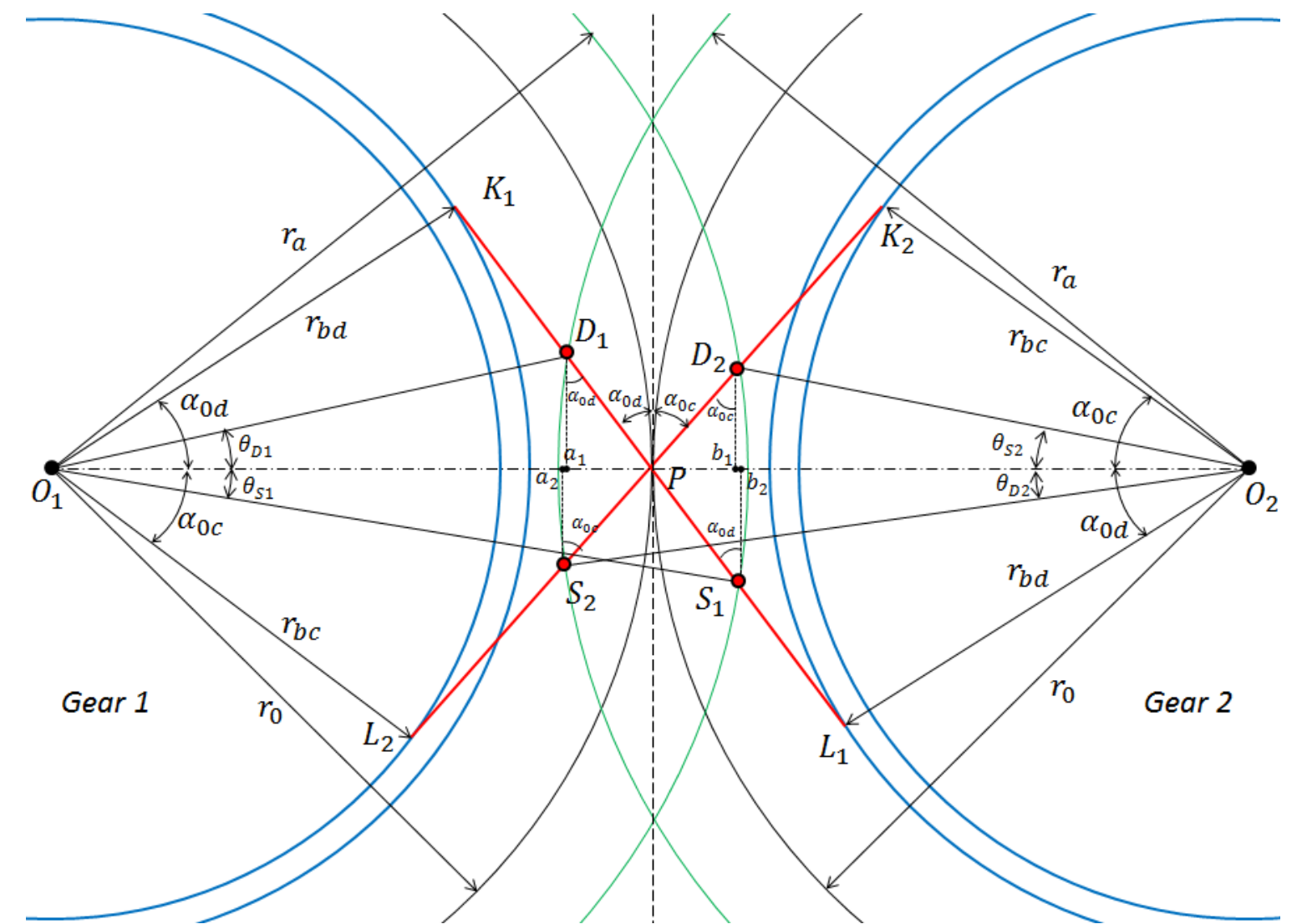

Figure 6: Meshing of the gears showing the lines of action (in red) and detail of the triangles required to calculate the angular location of the points of contact.

However, for a particular tooth pair, the active/working part of the line of action can be defined as the part of the line of action which is enveloped by the addendum circles of both the gears. Therefore, for the drive side, the active/working part of the line of action is defined as $D_{1} S_{1}$ and for the coast side as $D_{2} S_{2}$.

Let $l_{d}$ and $l_{c}$ be the lengths of the working part of the drive side and coast side lines of action respectively. With reference to Figure 6 , the line of action from the drive side and coast side can be written as,

$K_{1} S_{1}+D_{1} L_{1}=K_{1} L_{1}+l_{d}$ and $K_{2} S_{2}+D_{2} L_{2}=K_{2} L_{2}+l_{c}$.

Eq. (3) can be re-arranged as,

$l_{d}=K_{1} S_{1}+D_{1} L_{1}-K_{1} L_{1}$ and $l_{c}=K_{2} S_{2}+D_{2} L_{2}-K_{2} L_{2}$.

Considering the triangles $\Delta O_{1} K_{1} S_{1}$ and $\Delta O_{2} D_{1} L_{1}$ (henceforth in this document a triangle will be represented using the symbol $\Delta)$,

$K_{1} S_{1}=D_{1} L_{1}=\sqrt{r_{a}^{2}-r_{b d}^{2}}$.

Similarly considering $\Delta O_{2} K_{2} S_{2}$ and $\Delta O_{1} D_{2} L_{2}$,

$K_{2} S_{2}=D_{2} L_{2}=\sqrt{r_{a}^{2}-r_{b c}^{2}}$.

Considering $\triangle O_{1} K_{1} P$ and $\triangle O_{2} P L_{1}$,

$K_{1} P=P L_{1}=r_{0} \cdot \sin \alpha_{0 d}=r_{b 1} \cdot \tan \alpha_{0 d}$.

And with reference to $\triangle O_{2} K_{2} P$ and $\triangle O_{1} P L_{2}$,

$K_{2} P=P L_{2}=r_{0} \cdot \sin \alpha_{0 c}=r_{b 2} \cdot \tan \alpha_{0 c}$.

Substituting, Eqs. (5) - (8) into Eq. (4) yields the expressions for $l_{d}$ and $l_{c}$ as,

$l_{d}=2 \cdot \sqrt{r_{a}^{2}-r_{b d}^{2}}-2 \cdot r_{0} \cdot \sin \alpha_{0 d}$,

$l_{c}=2 \cdot \sqrt{r_{a}^{2}-r_{b c}^{2}}-2 \cdot r_{0} \cdot \sin \alpha_{0 c}$. 
Since the points, $K_{1}, D_{1}, S_{1}$ and $L_{1}$ are collinear,

$K_{1} P=K_{1} D_{1}+D_{1} P$.

Similarly, $K_{2}, D_{2}, S_{2}$ and $L_{2}$ are collinear, hence,

$K_{2} P=K_{2} D_{2}+D_{2} P$.

The pitch point ' $P$ ' divides the drive side line of action into two equal parts, therefore,

$D_{1} P=P S_{1}=\frac{D_{1} S_{1}}{2}=\frac{l_{d}}{2}=\sqrt{r_{a}^{2}-r_{b d}^{2}}-r_{0} \cdot \sin \alpha_{0 d}$,

$D_{2} P=P S_{2}=\frac{D_{2} S_{2}}{2}=\frac{l_{c}}{2}=\sqrt{r_{a}^{2}-r_{b c}^{2}}-r_{0} \cdot \sin \alpha_{0 c}$.

Considering $\Delta D_{1} a_{1} P$ in Figure 6 ,

$a_{1} P=D_{1} P \cdot \sin \alpha_{0 d}=\left(\sqrt{r_{a}^{2}-r_{b d}^{2}}-r_{0} \cdot \sin \alpha_{0 d}\right) \cdot \sin \alpha_{0 d}$,

$D_{1} a_{1}=D_{1} P \cdot \cos \alpha_{0 d}=\left(\sqrt{r_{a}^{2}-r_{b d}^{2}}-r_{0} \cdot \sin \alpha_{0 d}\right) \cdot \cos \alpha_{0 d}$.

Therefore,

$O_{1} a_{1}=O_{1} P-a_{1} P=r_{p}-\left(\sqrt{r_{a}^{2}-r_{b d}^{2}}-r_{0} \cdot \sin \alpha_{0 d}\right) \cdot \sin \alpha_{0 d}$

Hence, the angular position at which the drive side of a particular tooth of gear 1 comes in contact with the corresponding drive side of a tooth of gear $2, \theta_{D 1}$ can be represented as,

$\theta_{D 1}=\tan ^{-1}\left(\frac{D_{1} a_{1}}{O_{1} a_{1}}\right)=\tan ^{-1}\left(\frac{\left(\sqrt{r_{a}^{2}-r_{b d}^{2}}-r_{0} \cdot \sin \alpha_{0 d}\right) \cdot \cos \alpha_{0 d}}{r_{p}-\left(\sqrt{r_{a}^{2}-r_{b d}^{2}}-r_{0} \cdot \sin \alpha_{0 d}\right) \cdot \sin \alpha_{0 d}}\right)$.

In a similar fashion, the angular position at which the coast side of a particular tooth of gear 1 moves away from being in contact with the corresponding coast side of a tooth of gear $2, \theta_{S 2}$ can be represented as,

$\theta_{S 2}=\tan ^{-1}\left(\frac{a_{2} S_{2}}{O_{2} a_{2}}\right)=\tan ^{-1}\left(\frac{\left(\sqrt{r_{a}^{2}-r_{b c}^{2}}-r_{0} \cdot \sin \alpha_{0 c}\right) \cdot \cos \alpha_{0 c}}{r_{p}+\left(\sqrt{r_{a}^{2}-r_{b c}^{2}}-r_{0} \cdot \sin \alpha_{0 c}\right) \cdot \sin \alpha_{0 c}}\right)$.

After defining the angular positions at which both the sides of the same tooth (on gear 1 ) comes in contact and moves away from contact, it is easy to describe the angular range within which the TSV of gear 1 (drive) is trapped. For gear 1, this angular range is given by

$\theta_{T R 1}=\theta_{D 1}+\theta_{S 2}$.

Similarly for gear 2, the angular range within which the TSV of gear 2 is trapped between the points of contact be represented as,

$\theta_{T R 2}=\theta_{D 2}+\theta_{S 1}$.

Where $\theta_{D 2}$ and $\theta_{S 1}$ can be defined using a derivation similar to the ones described between Eqs. (11) - (19). Eqs. (20) - (21) hold only for the case of assuming dual flank contact configuration of the gears. However, for the case of gears operating with single flank contact configuration (as depicted in Figure $5(B)$ ) due to the presence of backlash between the gears, the consideration of the trapped TSV can be represented as,

$\theta_{T R}=\theta_{D 1}+\theta_{S 1}$.

The comparison between Eq. (22) with the Eqs. (20) - (21) highlights of the dual flank conditions permits to achieve larger intervals of trapped TSV, thus enhancing the range of of flow variation achievable by the proposed solution for VD-EGM.

\section{Analytical Determination of Geometric Displacement, Flow Rate and Input Torque}

With reference to the energy method described in [5-6] for the case of gears with symmetric teeth , a similar approach has been followed in this section to derive expressions suitable to study the displaced volume, the theoretical flow rate and the theoretical torque for the proposed VD-EGM concept.

\subsection{Theoretical Flow Rate}

Consider an EGM with symmetrical teeth in the configuration shown in Figure 7. The red gear profile shows the initial position of the gears and the black gear profile represents their final position after both gears rotate by a small angle $d \phi$. As shown in Figure 7, the pressure distribution around the gears is assumed with the pink and blue colors representing the regions of high pressure and low pressure respectively. This approximation is realistic for many EGMs [27] and permits to 
simplify some passages in the following derivations. However, the described method will still hold good even in case a gradual pressure variation is assumed around the gears.

Consider the pump shown in Figure 7, to be operating at a inlet pressure of $P_{\text {in }}=0$ and an outlet pressure of $P_{\text {out }}$. Therefore the pressure difference across the pump is $\Delta p=P_{\text {out }}$. Assuming, that the operating fluid of the machine is incompressible, the work done by the pump to displace a volume, $d v$ over a pressure barrier of $\Delta p$ can be written as,

$d W=d v \cdot \Delta p$

Hence, the amount of work/energy to be input to the pump, can be written as,

$d W=\left(M_{1}+M_{2}\right) \cdot d \phi$,

where $M_{1}$ and $M_{2}$, are respectively torques acting on gear 1 and 2, with respect to their axis of rotation and opposite to the direction of rotation. Taking the time derivate of eqs. (23) and (24) gives,

$\frac{d v}{d t} \cdot \Delta p=\left(M_{1}+M_{2}\right) \cdot \frac{d \phi}{d t}=Q \cdot \Delta p=\left(M_{1}+M_{2}\right) \cdot \omega$.

where $\omega$ is the angular velocity of gear 1 and $Q$ is the flow rate delivered by the pump Figure 7.

Considering the pressure distribution as shown in Figure 7, the torque acting on gear 1 can be written as [6],

$M_{1}=\left(\frac{r_{a}^{2}-r_{r}^{2}}{2}-\frac{r_{1}^{2}-r_{r}^{2}}{2}\right) \cdot b \cdot \Delta p=\left(\frac{r_{a}^{2}-r_{1}^{2}}{2}\right) \cdot b \cdot \Delta p$,

where, $b$ is the facewidth of the gears, $r_{1}$ is the distance $O_{1} C^{\prime}$ of the point of contact $C^{\prime}$ from the center of the gear $1, O_{1}$ as shown in Figure 7.

Similarly, the torque acting on gear 2 can be written as [6],

$M_{2}=\left(\frac{r_{a}^{2}-r_{2}^{2}}{2}\right) \cdot b \cdot \Delta p$.

Where, $r_{2}$ is the distance $\mathrm{O}_{2} \mathrm{C}^{\prime}$ of the point of contact $C^{\prime}$ from the center of gear 2, $\mathrm{O}_{2}$ as shown in Figure 7.

Substituting eqs. (26) and (27) into eq. (25) and re-arranging yields,

$Q=b \cdot \omega \cdot\left(\left(\frac{r_{a}^{2}-r_{1}^{2}}{2}\right)+\left(\frac{r_{a}^{2}-r_{2}^{2}}{2}\right)\right)$.

Eq. (28) provides an expression for calculating the flow rate, provided the information of $r_{1}$ and $r_{2}$ are readily available.

Equation (28) has also a geometric interpretation, which can be better explained in the following discussion. In order to make this geometric interpretation a little easier, it is assumed that the instantaneous points in the line of action, $l_{c}$ (which is the loci of the points of contact in the meshing zone of the gears), separates the high pressure from the low pressure at each angular position. Therefore, in the meshing zone, the region above line $O_{1} C^{\prime}$ for gear 1 and above line $O_{2} C^{\prime}$ for gear 2 are at high pressure and the regions below these lines are at low pressure. On the other hand, the line $O_{1} A^{\prime}$ for gear 1 and above line $\mathrm{O}_{2} \mathrm{~A}^{\prime}$ for gear 2 represent regions of high pressure and the regions below these lines are at low pressure.

With reference to Figure 7, consider the control volume, $\mathrm{O}_{1} \mathrm{O}_{2} B A O_{1}$ (also encompassed by the pink color), which basically represents the entire region in high pressure. Due to a small angular rotation of both the gears by an angle, $d \phi$, the control volume changes to $\mathrm{O}_{1} \mathrm{O}_{2} B^{\prime} A^{\prime} O_{1}$ (also encompassed by the pink color). The change in volume or displaced volume, $d v$ can be represented as,

$d v=$ volume $O_{1} O_{2} B^{\prime} A^{\prime} O_{1}-$ volume $O_{1} O_{2} B A O_{1}$. 


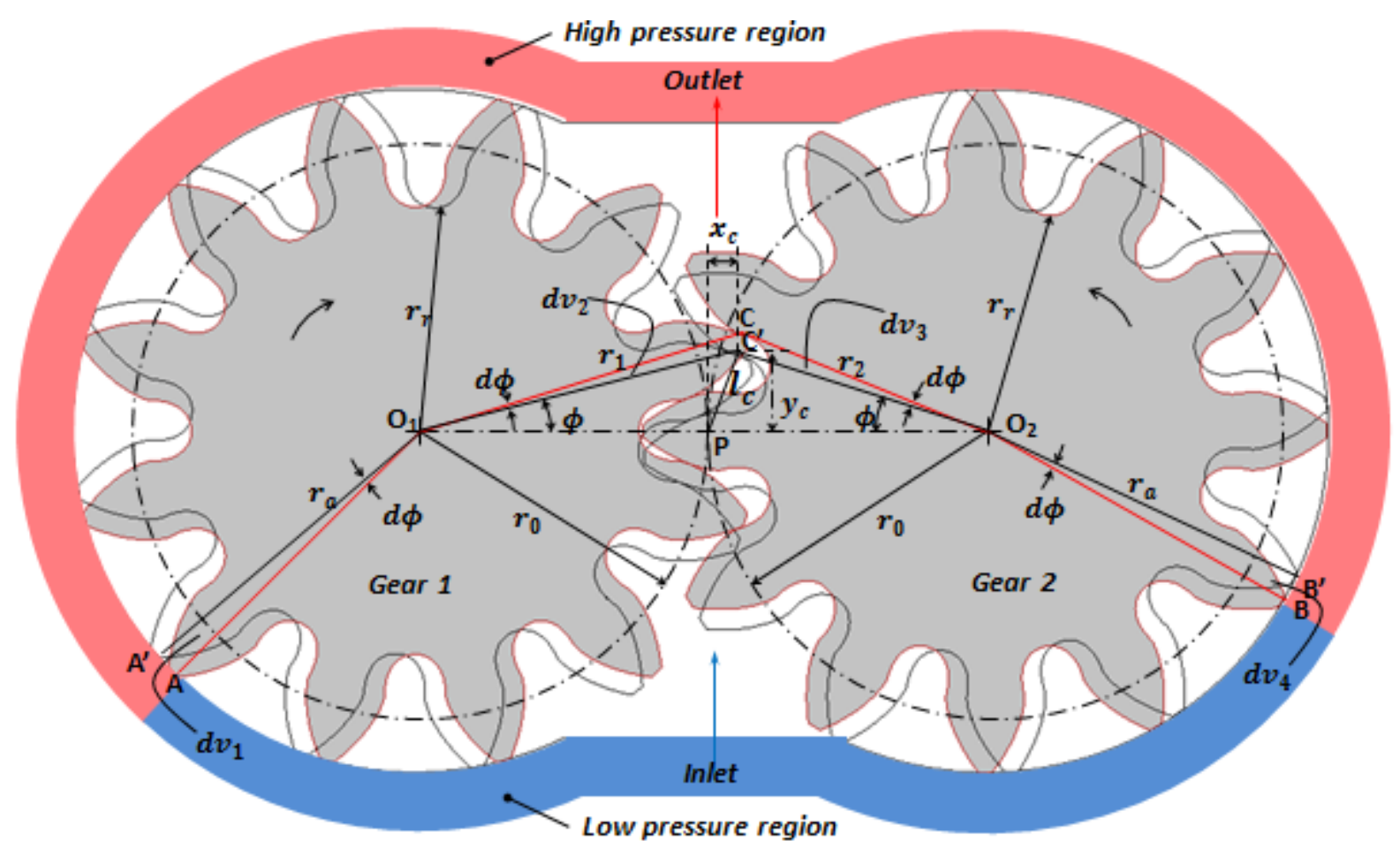

Figure 7: Determination of the displacement of the pump with symmetric teeth. [red] represents the initial position of the gears and [black] represents the final position of the gears.

In achieving this change in the control volume, the volumes, $d v_{1}$ and $d v_{4}$ enter the control volume, and the volumes, $d v_{2}$ and $d v_{3}$ leave the control volume as shown in Figure 7. Therefore, the displaced volume, $d v$ can be represented as, $d v=d v_{1}+d v_{4}-d v_{2}-d v_{3}$.

Since the angle through which the gears rotate are considered to be infinitesimally small, the volumes which enter and leave the control volume can be written as,

$d v_{1}=b \cdot \frac{r_{a}^{2}}{2} \cdot d \phi$

$d v_{2}=b \cdot \frac{r_{1}^{2}}{2} \cdot d \phi$

$d v_{3}=b \cdot \frac{r_{2}^{2}}{2} \cdot d \phi$

$d v_{4}=b \cdot \frac{r_{a}^{2}}{2} \cdot d \phi$

Substituting eqs. (31) - (34) in eq. (30) yields,

$d \nu=b \cdot \frac{r_{a}^{2}}{2} \cdot d \phi+b \cdot \frac{r_{a}^{2}}{2} \cdot d \phi-b \cdot \frac{r_{1}^{2}}{2} \cdot d \phi-b \cdot \frac{r_{2}^{2}}{2} \cdot d \phi$.

Re-arranging eq. (3.46 yields,

$d v=b \cdot\left(r_{a}^{2}-\frac{r_{1}^{2}}{2}-\frac{r_{2}^{2}}{2}\right) \cdot d \phi$

If the origin is translated to the pitch point $\mathrm{P}$, of the gears, the co-ordinates of the point $C^{\prime}$ can be represented by $x_{c}$ and $y_{c}$ as shown in Figure 7. The distance of point $\mathrm{C}$ from the origins of gears 1 and 2 can be written as [7],

$r_{1}^{2}=\left(r_{0}+x_{c}\right)^{2}+y_{c}^{2}=r_{0}^{2}+2 \cdot r_{0} \cdot x_{c}+x_{c}^{2}+y_{c}^{2}$,

$r_{2}^{2}=\left(r_{0}-x_{c}\right)^{2}+y_{c}^{2}=r_{0}^{2}-2 \cdot r_{0} \cdot x_{c}+x_{c}^{2}+y_{c}^{2}$.

Also from Figure $7, l_{c}$ can be written as,

$l_{c}^{2}=x_{c}^{2}+y_{c}^{2}$. 


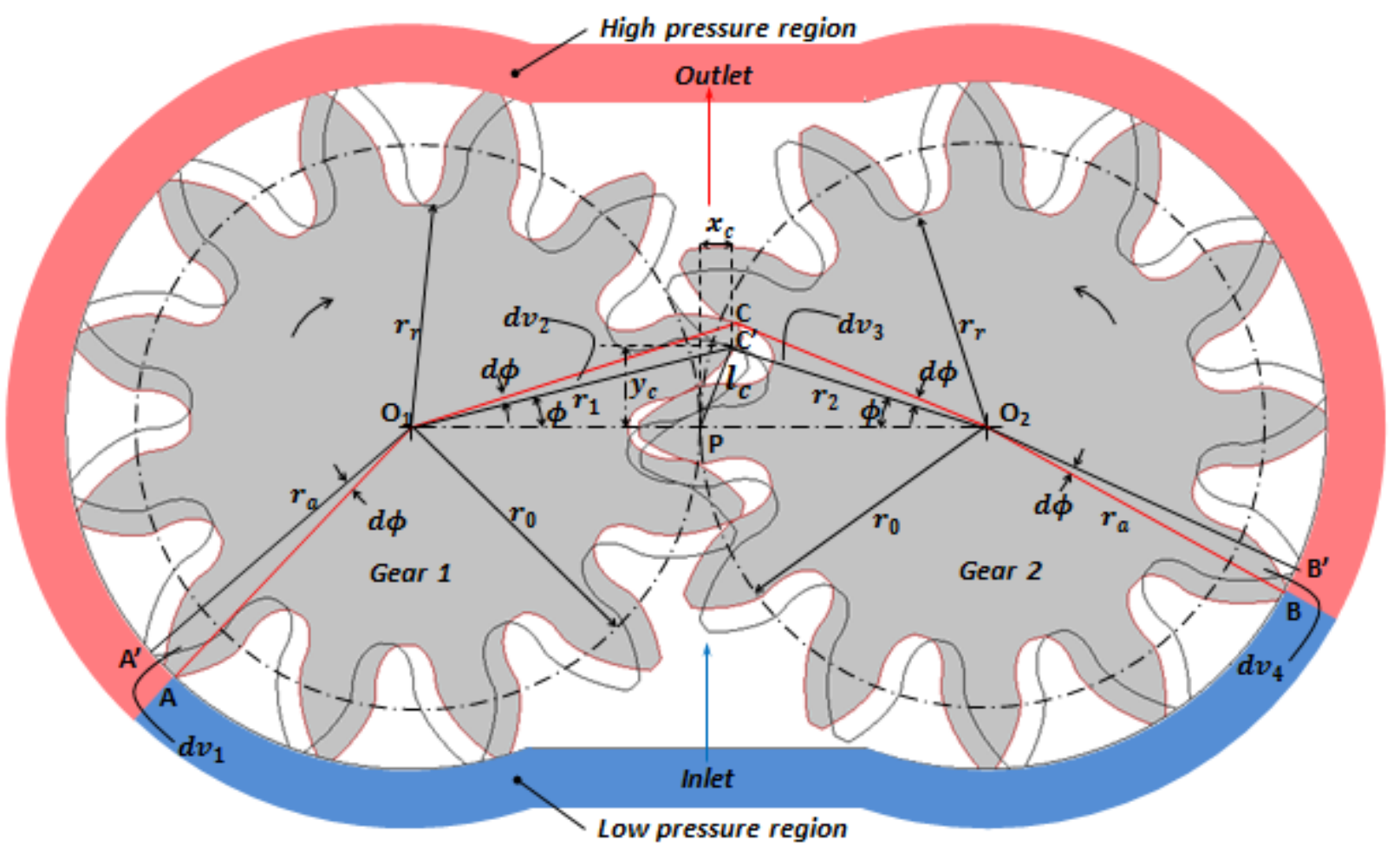

Figure 8: Determination of the displacement of the pump with asymmetric teeth showing the point of contact (C) on the coast side of the teeth. Red lines represent the initial position of the gears and black lines represent the final position of the gears.

Substituting eq. (39) in eqs. $(37,38)$ and re-arranging yields, [6-8], $d v=b \cdot\left(r_{a}^{2}-r_{0}^{2}-l_{c}^{2}\right) \cdot d \phi$.

It should also be noticed that $l_{c}$, varies with the angular position of the gears and hence it is a function of $\phi$.For small angle $\phi, l_{c}$ can be approximated as,

$l_{c}=r_{0} \cdot \phi \cdot \cos \alpha=r_{b} \cdot \phi$,

Where, $\alpha$ is the pressure angle of the symmetric gears considered and $r_{b}$ is the base radius of the gears.

It is important to remark that eq. (40) is valid under the assumption of symmetric gears since it depends only on one pressure angle. Therefore, when applying such an expression for deriving the displaced volume using asymmetric gears, the pressure angles corresponding to both the sides of the teeth need to be carefully incorporated into eq. (40) to have an accurate evaluation of the displaced volume.

Consider Figure 8, in which the meshing of asymmetric gears has been depicted in a similar fashion as in Figure 7. In Figure 8 , the point of contact has been represented on the coast side and hence the loci of the point of contact lie along the coast side line of action, $l_{c}$. On the other hand, the point of contact can be represented on the drive side and hence the loci of the point of contact lie along the drive side line of action, $l_{d}$ (not shown in figure). Due to the presence of two different pressure angles forming the asymmetric teeth, the angular position at which the coast side of a particular tooth of gear 1 comes in contact with the corresponding coast side of a tooth of gear 2, $\theta_{D 2}$ and the angular position at which the drive side of a particular tooth of gear 1 comes in contact with the corresponding drive side of a tooth of gear $2, \theta_{D 1}$ are different as explained in section 3. Hence the lines of action, $l_{c}$ and $l_{d}$ are different for any angular position $\phi$, as shown in Figure 8 . Since the number of teeth for both the gears are the same, it is safe to conclude that the displaced volume is an average of the volume displaced calculated using $l_{c}$ and $l_{d}$.

Therefore, for gears with asymmetric teeth, the displaced volume can be evaluated as an average of the displaced volume provided by the drive side and the coast side of the gear tooth. Hence, $d v$, can be represented as,

$d v=\frac{1}{2} \cdot b \cdot\left(r_{a}^{2}-r_{0}^{2}-l_{c}^{2}\right) \cdot d \phi+\frac{1}{2} \cdot b \cdot\left(r_{a}^{2}-r_{0}^{2}-l_{d}^{2}\right) \cdot d \phi$.

Rearranging, eq. (3.55) yields,

$d v=b \cdot\left(r_{a}^{2}-r_{0}^{2}\right) \cdot d \phi-\frac{1}{2} \cdot b \cdot\left(l_{c}^{2}+l_{d}^{2}\right) \cdot d \phi$. 
The instantaneous flow rate delivered by the pump with asymmetric gears can be written as,

$Q(\phi)=\frac{d v}{d t}=b \cdot\left(r_{a}^{2}-r_{0}^{2}\right) \cdot \frac{d \phi}{d t}-\frac{1}{2} \cdot b \cdot\left(l_{c}^{2}+l_{d}^{2}\right) \cdot \frac{d \phi}{d t}$.

Therefore,

$Q(\phi)=b \cdot\left(r_{a}^{2}-r_{0}^{2}\right) \cdot \omega-\frac{1}{2} \cdot b \cdot\left(l_{c}^{2}+l_{d}^{2}\right) \cdot \omega$,

For a small angle, $\phi$,

$l_{c}=r_{0} \cdot \phi \cdot \cos \alpha_{0 c}=r_{b c} \cdot \phi$,

$l_{d}=r_{0} \cdot \phi \cdot \cos \alpha_{0 d}=r_{b d} \cdot \phi$.

Substituting eq. (46-47) in eq. (45) yields,

$Q(\phi)=b \cdot\left(r_{a}^{2}-r_{0}^{2}\right) \cdot \omega-\frac{1}{2} \cdot b \cdot\left(r_{b c}^{2}+r_{b d}^{2}\right) \cdot \phi^{2} \cdot \omega$.

\subsection{Maximum Displacement of the VD-EGM}

Using the expression in eq.(48), the mean flow rate delivered at maximum displacement to the outlet can be written as, $Q_{m}^{\max }$,

$Q_{m}^{\max }=\frac{z}{\pi} \cdot \int_{-\frac{\pi}{2 \cdot z}}^{\frac{\pi}{2 \cdot z}}\left(b \cdot\left(r_{a}^{2}-r_{0}^{2}\right) \cdot \omega-\frac{1}{2} \cdot b \cdot\left(r_{b c}^{2}+r_{b d}^{2}\right) \cdot \phi^{2} \cdot \omega\right) d \phi$,

Notice that, in eq. (49) the integration has been performed within the limits within which the displacement chamber or the TSV changes its connection from the high pressure outlet to the low pressure inlet (based on the position of the simplified grooves shown in Figure 9). The integration limits represent the region within which the trapped TSV crosses the pressure barrier defined by the difference between outlet and inlet pressures, to be consistent with the assumption for eq.(23). The integrant is also multiplied by $\frac{z}{\pi}$, which corresponds to $2 \cdot z$ TSVs (twice the number of teeth displacement chambers due to the assumption of dual flank contact configuration) operating through a $2 \pi$ rotation of the gears.

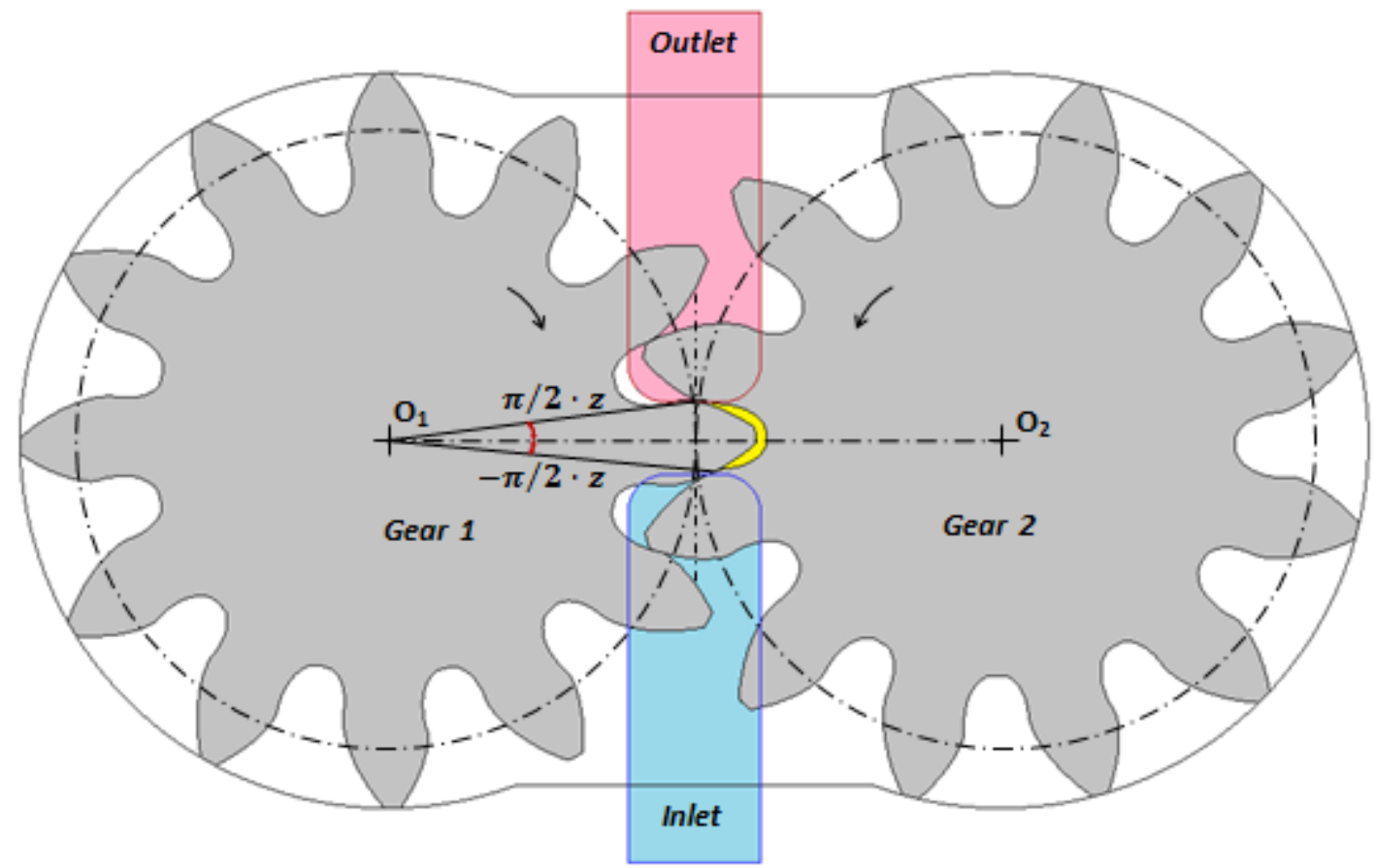

Figure 9: Position of the asymmetric gears and grooves showing the integration limits for maximum displacement. Integrating eq.(49), yields,

$Q_{m}^{\max }=b \cdot \omega \cdot\left(r_{a}^{2}-r_{0}^{2} \cdot\left(1+\frac{\pi^{2}}{24 \cdot z^{2}} \cdot\left(\cos {\alpha_{0 c}}^{2}+\cos \alpha_{0 d}{ }^{2}\right)\right)\right)$.

The mean value of the geometric maximum displacement for asymmetric gears can be evaluated using, 
$V^{\max }=\frac{Q_{m}^{\max }}{\omega / 2 \cdot \pi}=2 \cdot \pi \cdot b \cdot\left(r_{a}^{2}-r_{0}^{2} \cdot\left(1+\frac{\pi^{2}}{24 \cdot z^{2}} \cdot\left(\cos {\alpha_{0 c}}^{2}+\cos {\alpha_{0 d}}^{2}\right)\right)\right)$.

The total torque input to the shaft for maximum displacement can be written using eq. (25) as,

$M^{\max }=\frac{Q_{m}^{\max } \cdot \Delta p}{\omega}=b \cdot\left(r_{a}^{2}-r_{0}^{2} \cdot\left(1+\frac{\pi^{2}}{24 \cdot z^{2}} \cdot\left(\cos \alpha_{0 c}{ }^{2}+\cos \alpha_{0 d}{ }^{2}\right)\right)\right) \cdot \Delta p$.

\subsection{Minimum Displacement of the VD-EGM}

In a similar approach as considered in the previous section, the mean flow rate delivered at minimum displacement can be calculated. However, the integration limits for this case is more complicated than the previous case, due to the fact that the two different lines of action: the drive side $\left(l_{d}\right)$ and the coast side $\left(l_{c}\right)$ end at two different asymmetric locations represented by $\theta_{S 2}$ and $\theta_{S 1}$ respectively (as previously explained in Section 3), hence the integration limits have to be considered taking into account $\theta_{S 2}$ and $\theta_{S 1}$ separately. The integration limits for the drive side line of action is represented in Figure 10. A similar representation for the limits for the coast side line of action can also be made (not shown in figure).

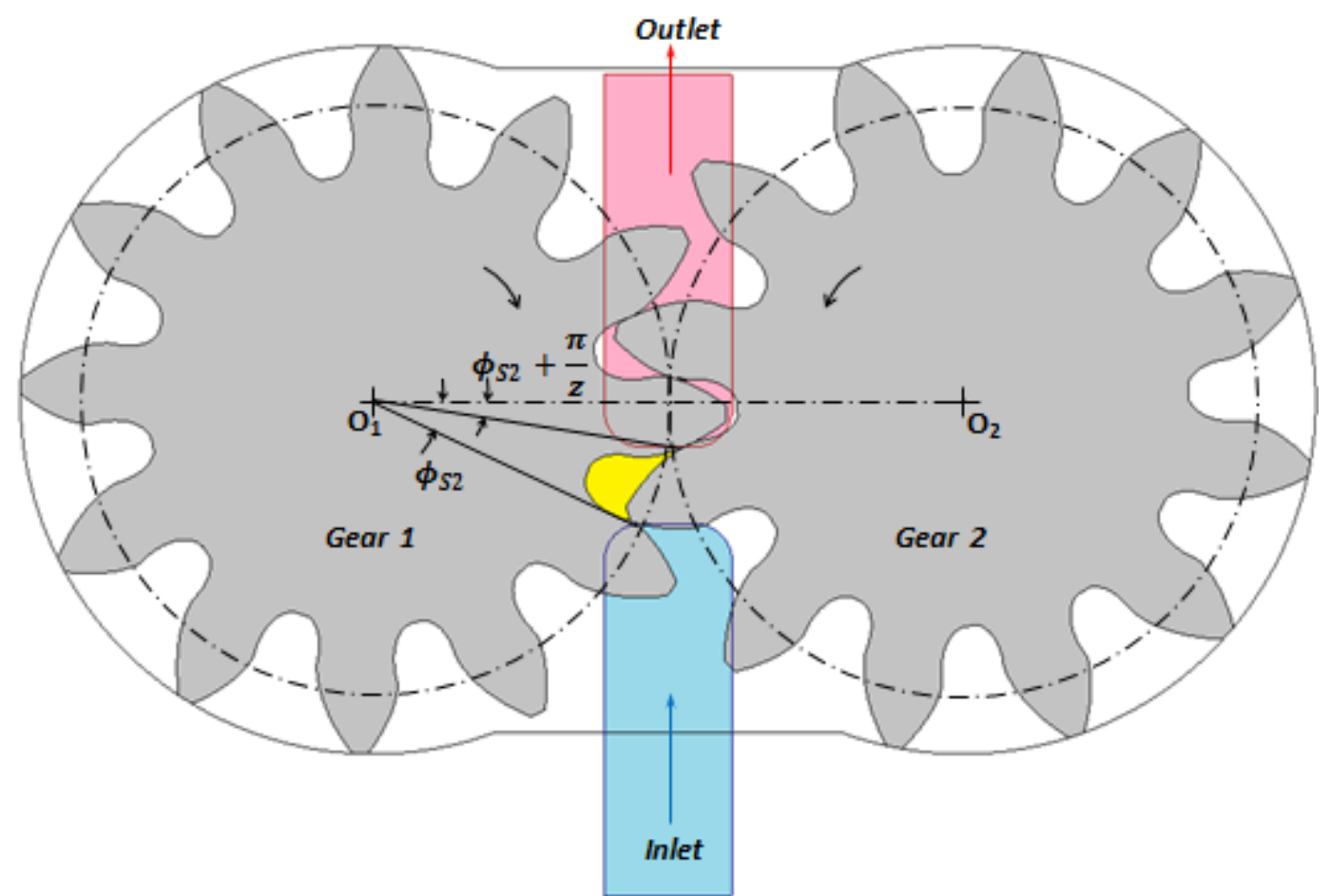

Figure 10: Position of the asymmetric gears and grooves showing the integration limits for minimum displacement.

Mathematically, the limits of integration considering the drive side line of action can be represented as,

$-\phi_{S 2} \leq \phi \leq-\phi_{S 2}+\frac{\pi}{z}$.

Similarly, the limits of integration considering the coast side line of action can be represented as,

$-\phi_{S 1} \leq \phi \leq-\phi_{S 1}+\frac{\pi}{z}$.

The mean flow rate delivered at minimum displacement can be expressed by re-arranging eq. (48) and applying the appropriate integration limits as,

$Q_{m}^{\min }=b \cdot \omega \cdot\left(r_{a}^{2}-r_{0}^{2} \cdot\left(1+\frac{\cos \alpha_{0 d^{2}}}{6} \cdot\left(\frac{\pi^{2}}{z^{2}}-3 \cdot \phi_{S 2} \cdot \frac{\pi}{z}+3 \cdot \phi_{S 2}^{2}\right)+\frac{\cos \alpha_{0 c}^{2}}{6} \cdot\left(\frac{\pi^{2}}{z^{2}}-3 \cdot \phi_{S 1} \cdot \frac{\pi}{z}+3 \cdot \phi_{S 1}^{2}\right)\right)\right)$.

The mean value of the geometric minimum displacement for asymmetric gears can be evaluated using,

$V^{\min }=\frac{Q_{m}^{\min }}{\omega / 2 \cdot \pi}$ 


$$
=2 \cdot \pi \cdot b \cdot\left(r_{a}^{2}-r_{0}^{2} \cdot\left(1+\frac{\cos \alpha_{0 d^{2}}}{6} \cdot\left(\frac{\pi^{2}}{z^{2}}-3 \cdot \phi_{S 2} \cdot \frac{\pi}{z}+3 \cdot \phi_{S 2}^{2}\right)+\frac{\cos \alpha_{0 c}{ }^{2}}{6} \cdot\left(\frac{\pi^{2}}{z^{2}}-3 \cdot \phi_{S 1} \cdot \frac{\pi}{z}+3 \cdot \phi_{S 1}^{2}\right)\right)\right)
$$

The total torque input to the shaft for minimum displacement can be written as,

$M^{\min }=\frac{Q_{m}^{\min } \cdot \Delta p}{\omega}=b \cdot\left(r_{a}^{2}-r_{0}^{2} \cdot\left(1+\frac{\cos \alpha_{0 d^{2}}}{6} \cdot\left(\frac{\pi^{2}}{z^{2}}-3 \cdot \phi_{S 2} \cdot \frac{\pi}{z}+3 \cdot \phi_{S 2}^{2}\right)+\frac{\cos \alpha_{0 c}{ }^{2}}{6} \cdot\left(\frac{\pi^{2}}{z^{2}}-3 \cdot \phi_{S 1} \cdot \frac{\pi}{z}+3 \cdot\right.\right.\right.$

$\left.\left.\left.\phi_{S 1}^{2}\right)\right)\right) \cdot \Delta p$

Based on the expressions derived previously for maximum and minimum displacement, the resultant reduction in displacement can be written as,

$\beta=\frac{V^{\min }}{V^{\max }}$.

\section{Analytical Determination of Non-Uniformity in Flow Rate and Input Torque}

The flow non-uniformity (flow ripple) produced during the operation of the machine can be analytically as detailed in this section.

\subsection{Maximum Displacement}

In order to understand the non-uniformity of the flow rate delivered at the outlet, both the maximum and the minimum values of the instantaneous flow rate need to be determined. For the configuration of grooves to attain maximum displacement, eq. (49) is applicable to the region represented by,

$-\frac{\pi}{2 \cdot z} \leq \phi \leq \frac{\pi}{2 \cdot z}$

Within this region, the maximum value of the instantaneous flow rate is achieved when $\phi=0$ and the minimum value of the instantaneous flow rate is achieved when $\phi=\frac{\pi}{2 \cdot z}$.

Hence, the non-uniformity in the flow rate (or outlet flow pulsations) for maximum displacement can be represented as the difference between the maximum and minimum values of the instantaneous flow rate as,

$\delta_{Q}^{\max }=Q_{i, \max }^{\max }-Q_{i, \min }^{\max }=\frac{1}{8} \cdot b \cdot\left(r_{b c}^{2}+r_{b d}^{2}\right) \cdot{\frac{\pi^{2}}{z^{2}}}^{2} \cdot \omega$.

Similar to the non-uniformity in the flow rate, non-uniformity of input shaft torque (or input shaft torque pulsations) can be represented as,

$\delta_{M}^{\max }=\frac{\delta_{Q}^{\max } \cdot \Delta p}{\omega}=\frac{1}{8} \cdot b \cdot\left(r_{b c}^{2}+r_{b d}^{2}\right) \cdot \frac{\pi^{2}}{z^{2}} \cdot \Delta p$.

\subsection{Minimum Displacement}

Considering the limits of integration as shown in eq. (56), the maximum value of the instantaneous flow rate for minimum displacement configuration can be calculated by substituting $\phi=-\phi_{S 2}$ and $\phi=-\phi_{S 1}$ respectively in the drive side and coast side line of action in eq. (48).

Therefore, the non-uniformity in the flow rate (or outlet flow pulsations) for minimum displacement can be represented as the difference between the maximum and minimum values of the instantaneous flow rate as,

$\delta_{Q}^{\min }=Q_{i, \max }^{\min }-Q_{i, \min }^{\min }=\frac{1}{2} \cdot b \cdot\left(r_{b c}^{2} \cdot\left(\frac{\pi^{2}}{z^{2}}-2 \cdot \phi_{S 1} \cdot \frac{\pi}{z}\right)+r_{b d}^{2} \cdot\left(\frac{\pi^{2}}{z^{2}}-2 \cdot \phi_{S 2} \cdot \frac{\pi}{z}\right)\right) \cdot \omega$.

Comparing, eq. (60) and (62), the difference between the non-uniformity between the maximum and minimum displacement can be written as,

$\delta_{Q}^{\min }-\delta_{Q}^{\max }=\frac{3}{8} \cdot b \cdot\left(r_{b c}^{2}+r_{b d}^{2}\right) \cdot{\frac{\pi}{Z^{2}}}^{2} \cdot \omega+b \cdot\left(r_{b c}^{2} \cdot \phi_{S 1}+r_{b d}^{2} \cdot \phi_{S 2}\right) \cdot \frac{\pi}{Z} \cdot \omega$.

It can be clearly seen that the non-uniformity in the flow rate is higher at minimum displacement than that aximum displacement, which is inherent to the concept with which displacement variation is achieved. Therefore, the machine is expected to operate at a higher level of fluid borne noise at minimum displacement as compared to that of maximum displacement.

Similar to the non-uniformity in the flow rate, non-uniformity of input shaft torque (or input shaft torque pulsations) can be represented as, 
$\delta_{M}^{\min }=\frac{\delta_{Q}^{\min } \cdot \Delta p}{\omega}=\frac{1}{2} \cdot b \cdot\left(r_{b c}^{2} \cdot\left(\frac{\pi^{2}}{z^{2}}-2 \cdot \phi_{S 1} \cdot \frac{\pi}{z}\right)+r_{b d}^{2} \cdot\left(\frac{\pi^{2}}{z^{2}}-2 \cdot \phi_{S 2} \cdot \frac{\pi}{z}\right)\right) \cdot \Delta p$

In a similar manner to the non-uniformity in flow rate, the input shaft torque pulsations at minimum displacement are higher than that at maximum displacement.

\section{Analytical Results}

To validate the analytical expressions derived in this paper, an existing design for the gears suitable for the VD-EGM concept is considered. This design of the asymmetric gears was previously numerically optimized and presented by the authors in [3]. The profile of these gears resulted from an entirely numerical based method used to optimize asymmetric gears along with the grooves in the lateral bushings simultaneously. The optimization was based on a multi-objective genetic algorithm that uses HYGESim (HYdraulic GEar machines Simulator) to evaluate the performance of every considered design. HYGESim has been developed and validated by the authors' research team over the past several years [27-29], and it permits to take into considerations the detailed geometry of the unit; the effects of fluid compressibility and leakages as well as the radial micro-motions of the gears. The optimization algorithm varied the parameters of the tooth profile in order to satisfy a set of objective functions given by: minimization of flow ripple, minimization of internal pressure overshoots and localized cavitation; maximization of the volumetric efficiency; maximization of the range of displacement reduction. The details of the optimization problem and its implementation can be found in [3].

Table 1: Design parameters for the optimal design of the gears.

\begin{tabular}{|l|l|}
\hline Number of teeth & 16 \\
\hline Facewidth & $17.0 \mathrm{~mm}$ \\
\hline Drive pressure angle & $9.50^{\circ}$ \\
\hline Coast pressure angle & $5.00^{\circ}$ \\
\hline Addendum diameter & $38.34 \mathrm{~mm}$ \\
\hline Minor/root diameter & $25.12 \mathrm{~mm}$ \\
\hline Maximum displacement & $11.90 \mathrm{cc} / \mathrm{rev}$ \\
\hline Minimum displacement & $8.09 \mathrm{cc} / \mathrm{rev}$ \\
\hline Minimum displacement (\%) & 68 \\
\hline
\end{tabular}

The optimal design of the gears which provided maximum reduction in displacement, while maintaining all the other performance parameters at an optimum is shown in the Table 1 . The optimal design is atypical as compared to standard gears, particularly the pressure angles are low as well as the fillet part of the tooth are long as seen in Figure 11(A). The structural strength of the design was verified using suitable constraints as explained in [3] and experimental validations supporting the working of the design have been reported in $[3,4]$. The main reason behind the peculiarity of the presented design is that it permits to achieve an extended region of trapped tooth space volume, which is necessary for achieving a larger reduction in displacement. It is observed that the optimal design chosen is capable of offering a remarkable range of variation of displacement for $100 \%$ ( $\max$ ) to $68 \%(\mathrm{~min})$. This range was partially limited by the choice of generating gears able to be used in a casing/bearing set of a commercial unit to facilitate experiments. Therefore, the flow rate range could have been higher if some constraints about the gears (facewidth, inter-axis distance - and hence the pitch diameter. outer radius) were relaxed [24].

The optimal design of the gears and the optimal grooves designs and the position of the slider in the lateral bushings for achieving maximum and minimum displacement are shown in Figure 11. Notice that a particular "two-winged" structure of the grooves was considered for the optimization (compared to the simplified rectangular grooves used for the derivation), to determine an optimal design of the grooves can also ensure minimal internal pressure overshoots and localized cavitation effects during the transition of TSV from/to the low pressure and high pressure regions while maintaining maximum volumetric efficiency at different levels of displacement [3]. 


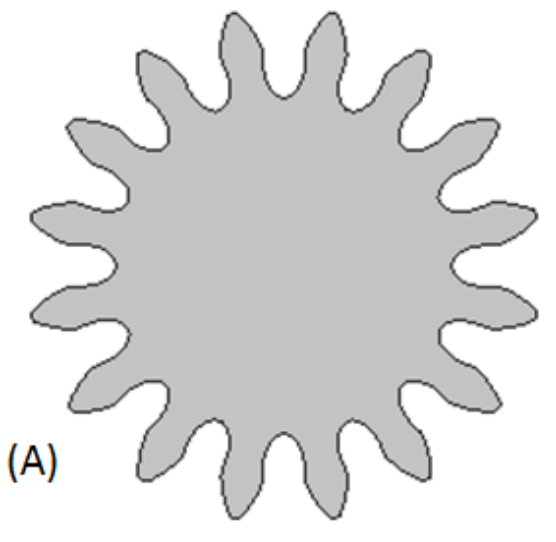

(B)

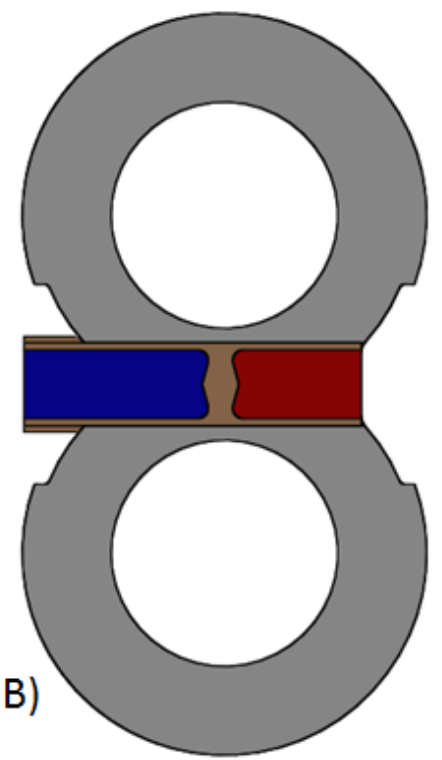

(C)

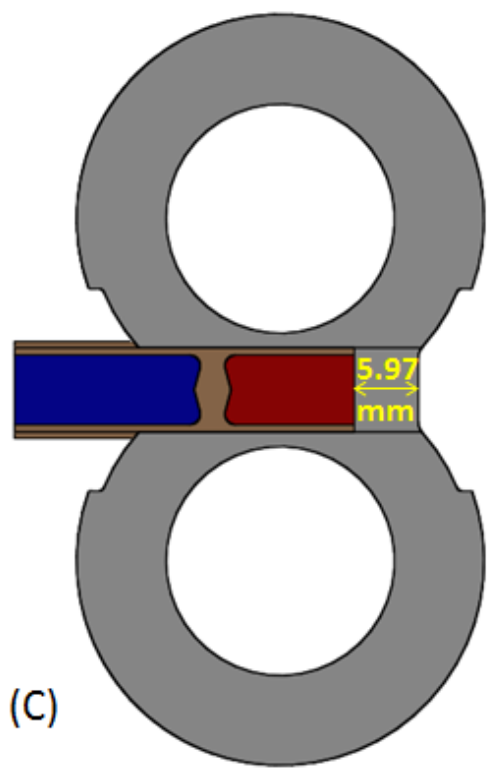

Figure 11: (A) Optimal design of the gears for variable displacement; (B) Optimal design of the grooves in the slider, with the slider positioned for achieving maximum displacement; (C) Optimal design of the grooves in the slider, with the slider positioned for achieving minimum displacement.

The values of the displacements were calculated numerically using the HYGESim simulation tool. In order to have a validation of the evaluations made by the analytical expressions derived in previous sections, eqs.(51), (56) and (58) need to be recalled. By substituting the geometric parameters pertaining to the gears (shown in The details of the optimization problem and its implementation can be found in [3].

Table 1 ) into eq.(51) and eq.(56), yields the following values, $V^{\max }=11.69^{c C} /$ rev, $V^{\min }=8.01^{\mathrm{cc}} / \mathrm{rev}$.

Moreover, the minimum displacement can be expressed in a percentage format as, $\beta=\frac{V^{\max }}{V^{\min }}=68.55 \%$

Comparing the values from eq. (65-67) with the values of maximum and minimum displacement from The details of the optimization problem and its implementation can be found in [3].

Table 1, it can be seen that the values predicted by the geometrical model is very similar to the ones predicted by the analytical equation and hence proving the validity of the equations and the model.

Detailed analyses of the optimized VD-EGM design were performed using HYGESim. Particularly, the main performance features such as the flow ripple, input shaft torque were analyzed for several operating conditions. The simulated flow rate at the delivery for a representative operating condition is shown in Figure 12(A). It can be seen that the flow rate at reduced displacement has reduced proportionally (68\%) to that at full displacement. This proves that the VD-EGM is capable of providing lower flow rates at reduced displacement. Even though the design optimization process considered the performance at both max and min displacement, the flow oscillations at min displacement, $(\beta=68 \%)$ is seen to be higher than that at max displacement for all the operating conditions. This difference in behavior was expected based on the analytical derivations which were previously made in section 4, wherein a higher non-uniformity of flow rate was predicted analytically at min displacement. As evidently visible from Figure 12(B), the tooth space pressure for min displacement ( $\beta=68 \%)$ extends for a larger time period as depicted by the red curves, because the TSVs are connected to the delivery/outlet groove for a larger time period to bring about a reduction in displacement.

A simplified representation of the implementation for manual setting of displacement is depicted in Figure 13(A). The slider is placed in the slot on the bearing block as shown in Figure 13(B). The position of the slider can be changed with the help of 
the bolt which connects to the slider through a connecting rod. The design of the slider should take into consideration its proper seating in the bearing block ensuring that no sharp edges come in contact with the gears rotation. It is also important to notice that the implementation of the slider introduces and additional leakage interface between the inlet and the outlet environment through the gap between the slider and the bearing block. Therefore, the actual flow rate will be lesser than that predicted by HYGESIM in Figure 12(A). The gap at the interface allows for a fluid film that will ensure that a pressure distribution similar to the one in the meshing zone facing the gears is present, thus ensuring no stiction between the slider and the bearing block. The authors implemented a pressure compensated type of design which was prototyped and successfully tested as described in [4]
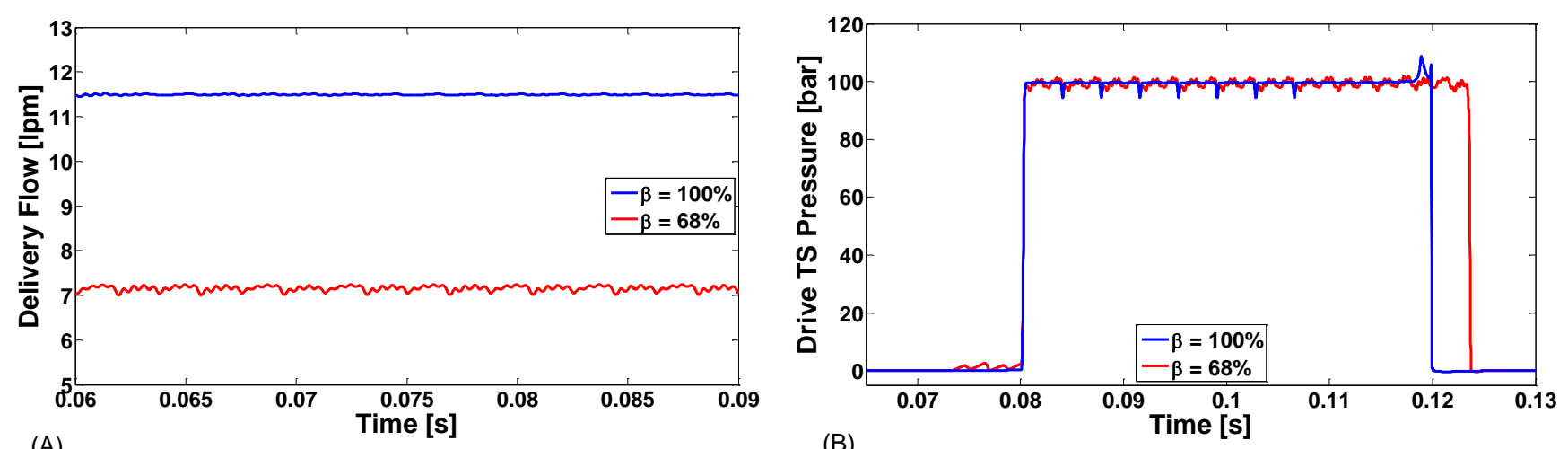

Figưre 12: (A) Delivery flow (B) Tooth space pressure at max. $(\beta \stackrel{(B)}{=} 100 \%)$ and $\min .(\beta=68 \%)$ displacement for $1000 \mathrm{rpm}$ $100 \mathrm{bar}$

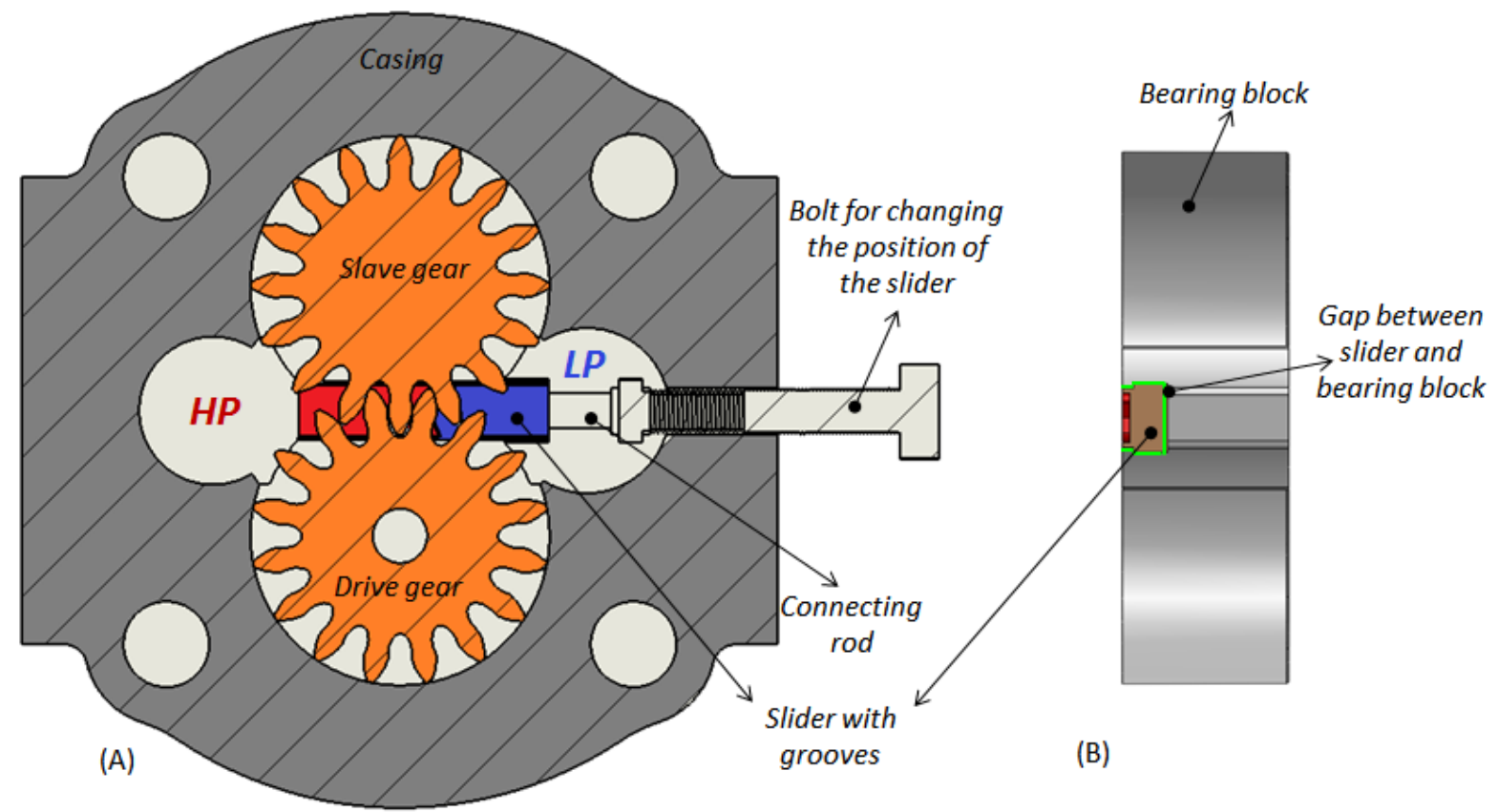

Figure 13: (A) Simplified representation of manual setting of displacement. (B) Detail of bearing block and slider assembly.

\section{Numerical Analysis of Force and Torque Generation in the Proposed Concept for VD-EGM}

The HYGESim simulation tool was also used to numerically predict the resultant radial forces and hence the shaft torque acting on the gears based on the pressure distribution of the displaced fluid. These results permit to show that the prolonged pressurization of the gears, for the min displacement condition, lead to a reduction in the shaft torque.

The numerical approach used by HYGESim, better described in [27], can be illustrated with the Figure 14. In particular, for every tooth space surface the pressure evaluated by the fluid dynamic model is used to calculate $F_{x}$ and $F_{y}$ forces, by properly considering the projected areas of pressure influence (areas $A$ in Figure 14). By summing up the contributions of 
every tooth space surface of each gear, the total radial force due to the pressure loading is found. In order to find the shaft torque, the moment arm of every force component is calculated at every angular position of the gears ( $x$ and $y$, in Figure 14). By assuming an equilibrium condition (constant speed) for the slave gear, a contact force is determined to balance the torque. By assuming the equilibrium condition for the drive gear, the shaft torque is determined.

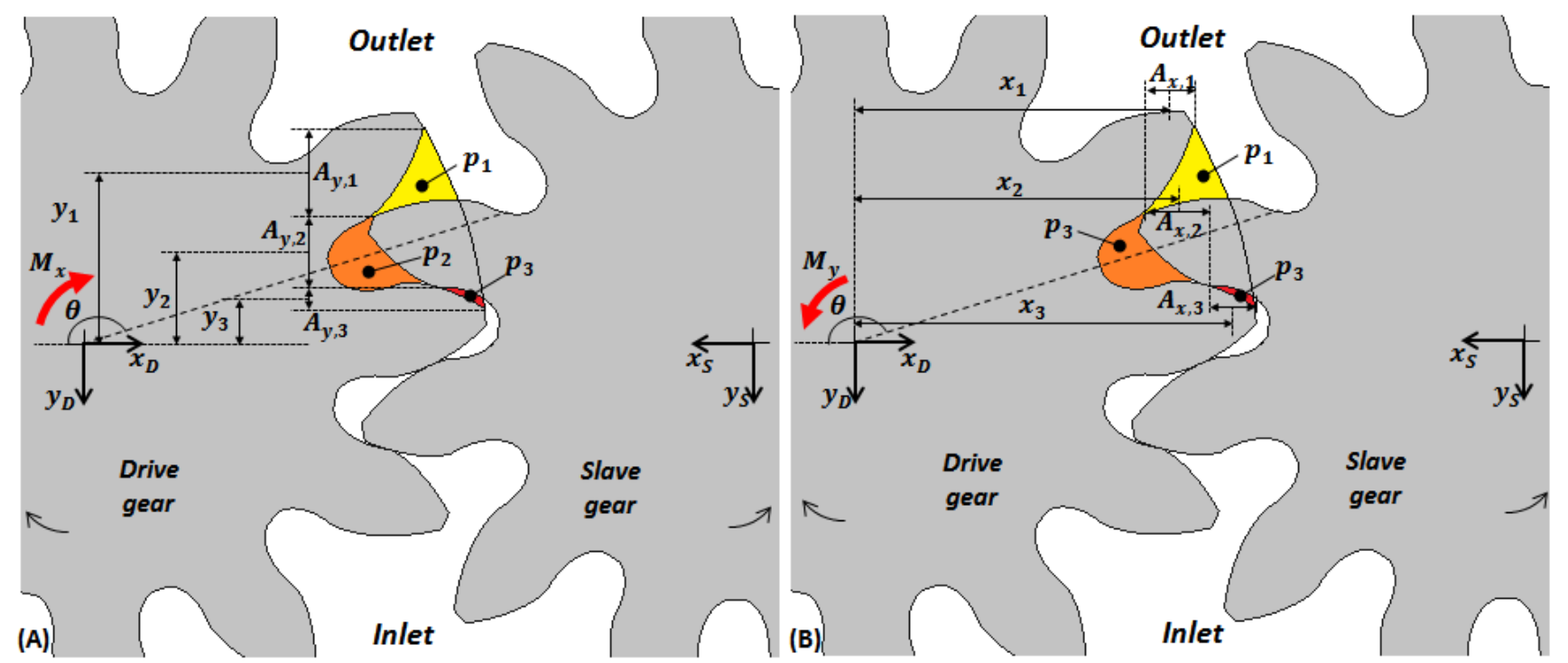

Figure 14: Subdivision of the tooth space surface between adjacent teeth on a gear. (A) Projected areas for calculation of forces along $x$ direction; (B) Projected areas for calculation of forces along y direction.
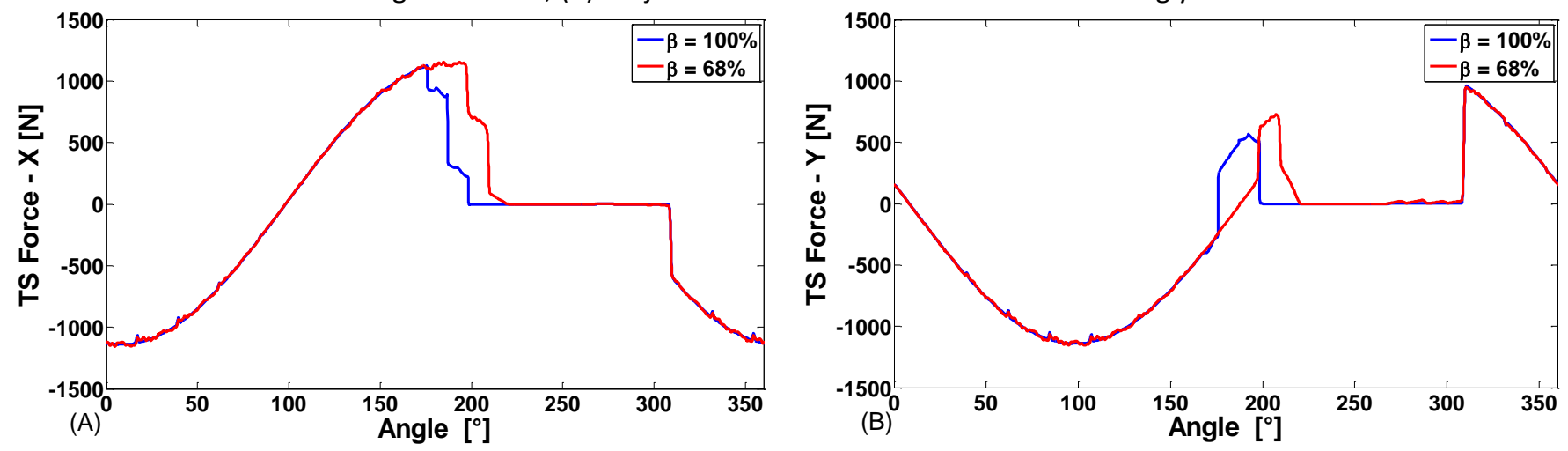

Figure 15: Tooth space force in (A) $x$ direction; (B) y direction for $1000 \mathrm{rpm} 100 \mathrm{bar}$

As an example of the calculation, Figure 15 shows the tooth space $F_{x}$ and $F_{y}$ contribution over the entire $360^{\circ}$ rotation of the gears (angular convention as shown in Figure 14) for a determined operating condition. From these figures it can be clearly noticed the difference between the forces at max and min displacement, due to the change in the pressure distribution obtained by the different positons of the slider. The calculations of the moments terms associated with such $F_{x}$ and $F_{y}$ forces are shown in Figure 16(A and B). Also for the moments a difference is noticeable when the position of the slider changes. 

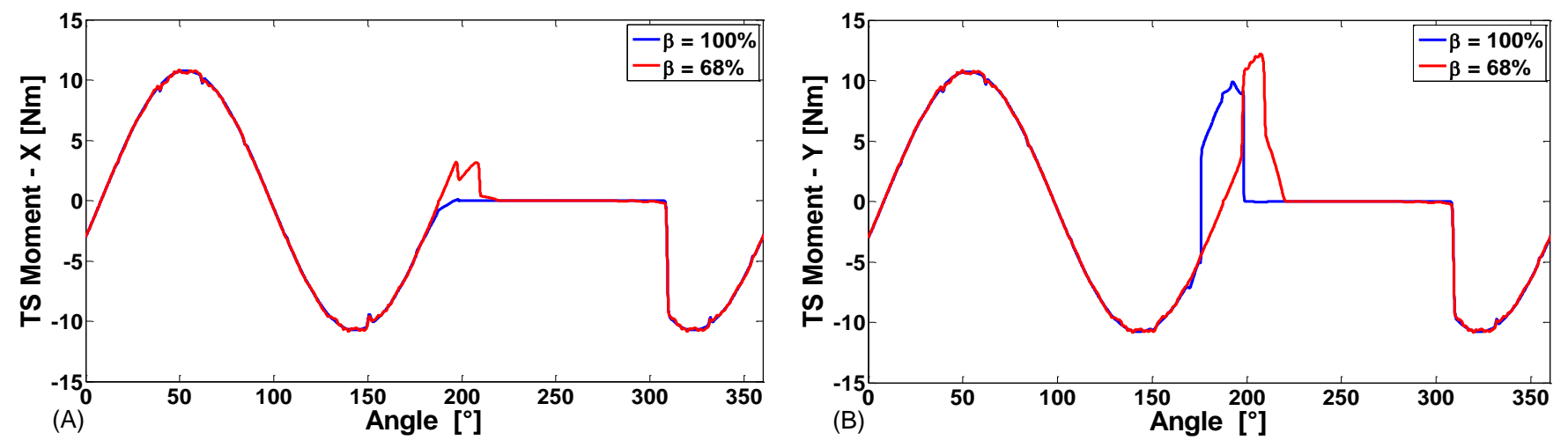

Figure 16: Tooth space moment due to force in (A) $x$ direction; (B) y direction for 1000rpm 100bar
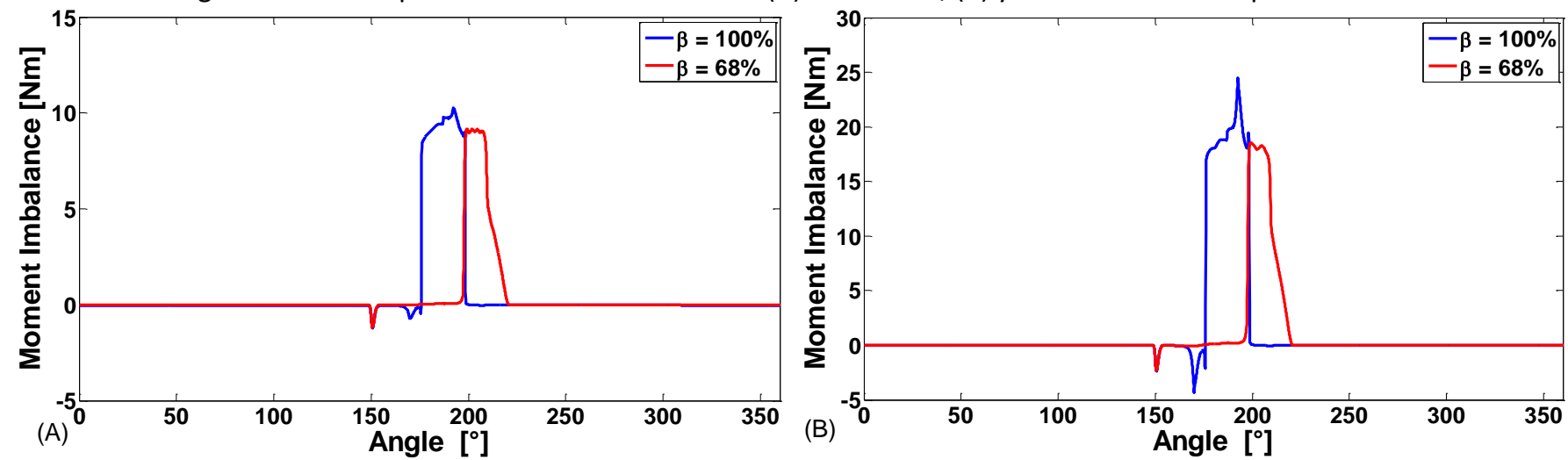

Figure 17: Moment imbalance for (A) 1000 rpm 100 bar; (B) 2000rpm 200bar.

Since the moments $M_{x}$ and $M_{y}$ act in opposite directions about the center of the considered gear (as shown in Figure 14), the moment/torque required to transfer the fluid in the considered tooth space for a complete revolution of the gear can be written as,

$M_{\text {net }}=M_{y}-M_{x}$

This net torque per single tooth space, for the cases of max and min displacements is shown in Figure 17 for two different operating conditions. It is evident from the figures that the area under the curve for min displacement is smaller than that at max displacement.

In order to better understand the difference in torque, an estimate of the energy consumption can be made by calculating the area under the moment imbalance curve. This value can be expressed as,

$E=\int_{0}^{2 \cdot \pi} M_{n e t} \cdot d \theta$.

Table 2: Summary of the estimate of energy consumption for max and min displacement.

\begin{tabular}{|l|l|l|l|}
\hline $\begin{array}{c}\text { Operating } \\
\text { Condition }\end{array}$ & \multicolumn{1}{|c|}{$\boldsymbol{E}_{\text {max }}$} & \multicolumn{1}{|c|}{$\boldsymbol{E}_{\text {min }}$} & \multicolumn{1}{c|}{$\frac{\boldsymbol{E}_{\min }}{\boldsymbol{E}_{\max }}$} \\
\hline $1000 \mathrm{rpm} 100 \mathrm{bar}$ & 193.25 & 132.66 & 0.68 \\
\hline $2000 \mathrm{rpm} 200 \mathrm{bar}$ & 410.02 & 266.70 & 0.65 \\
\hline
\end{tabular}

Table 2 shows the summary of the calculated estimates of energy using eq. (69). As can be seen from the ratio of the estimates, for $1000 \mathrm{rpm} 100 \mathrm{bar}$ case, the model predicts that at min displacement it consumes only $68 \%$ of the energy at max displacement. However, for 2000rpm 200bar case, there is an even further reduction in the energy consumption; this is due to the fact that there is a higher pressure peak (as compared to a negligible peak for 1000rpm 100bar case) is predicted by HYGESim in the tooth spaces during the meshing zone. Hence, numerically it has been proved that at min displacement there is reduction of torque and hence energy while considering a single tooth space surface. 
By symmetry the analysis can be extended to consider all the tooth spaces in both the gears and hence a $\sim 68 \%$ reduction in torque can be seen at min displacement due to the overall lower total force acting on the gears.

\section{Experimental Results}

A proof of concept prototype based on the gears/slider of Figure 11 was realized and tested at the authors' research center to support the research on the proposed concept of the VD-EGM. Details on the prototype and of the experimental setup were previously published in [3]. However, the focus of this past work was not on the analytical study of the VD-EGM concept. For this reason, in this section some of these results are recalled, followed by the addition of new test results, in light of supporting the validity of the analytical formulation proposed for studying the VD-EGM concept.

Flow Rate - 1000rpm

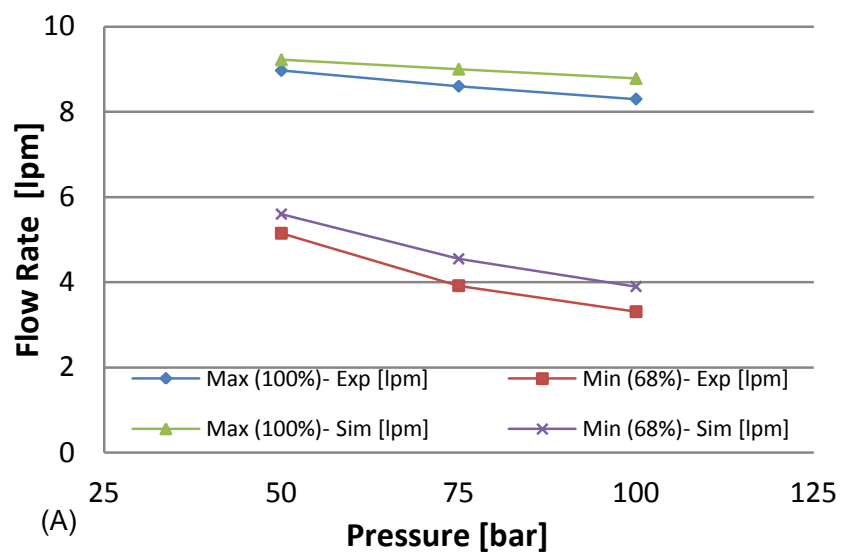

Torque - 1000rpm

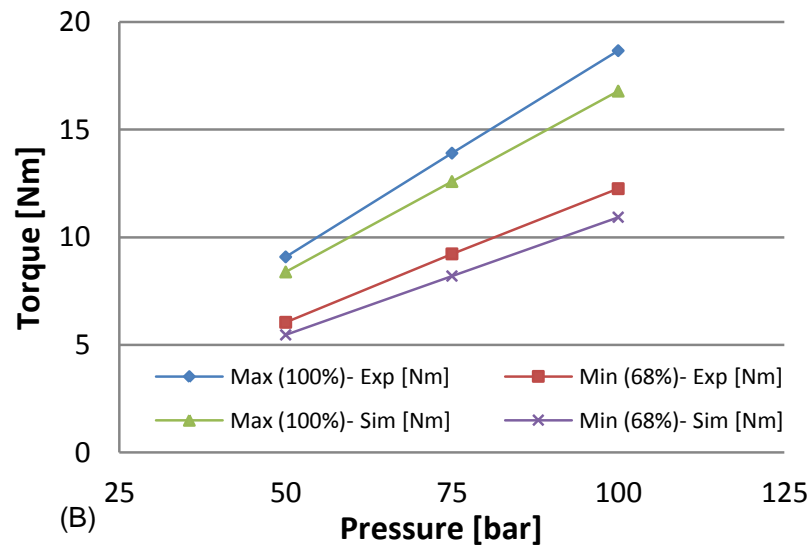

Figure 18: Validation for (A) Flow rate and (B) Input torque for $1000 \mathrm{rpm}$.

It can be seen from Figure 20(A) that the flow rate proportionally $(68 \%)$ reduces at min displacement as compared to those at full or max displacement. The simulated data matches very closely with that of the measurements. The minor discrepancy between the experimental and simulated data can be attributed to the imperfections in the machining of the gears as well as the simplifications in the model for the calculation of the leakages within the pump [3]. Approximately $32 \%$ reduction in torque is obtained for min displacement as shown in Figure 20(B). The simulations under predict the input shaft torque because the some important torque loss contributions were not taken into account into the analysis (in particular the shear losses in the internal lubricating gaps; frictional losses due to the sliding of the gear teeth with each other).
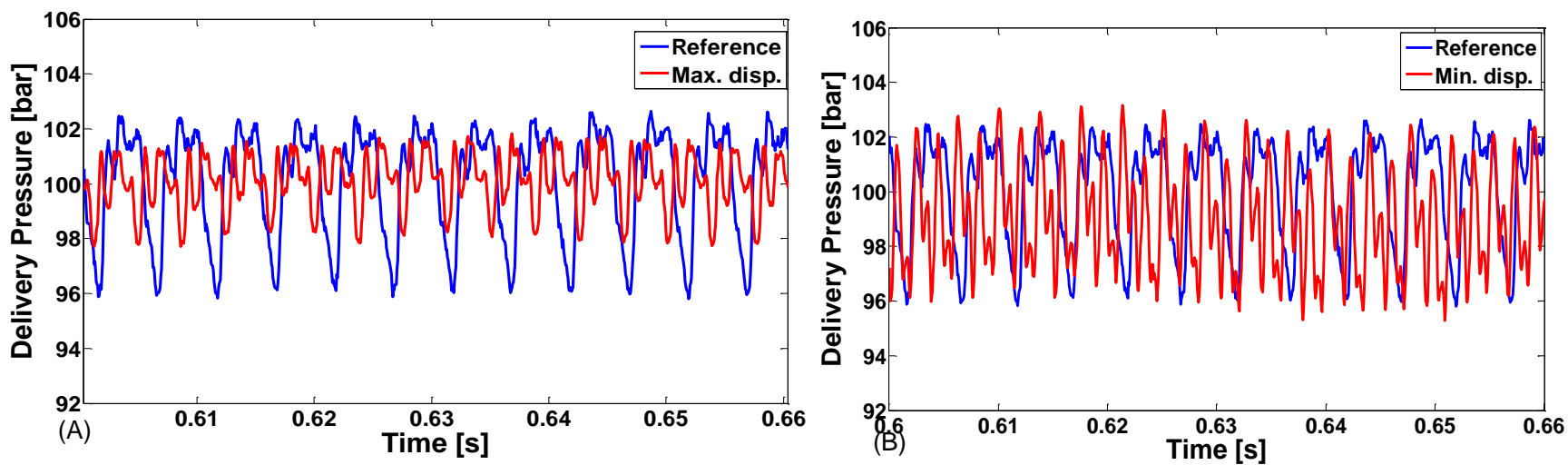

Figure 19: Delivery pressure ripple at 1000rpm, 100bar for the reference and (A) max displacement; (B) min displacement.

The pressure ripple at the delivery of the pump was also measured for a wide range of operating conditions, to study the effect of displacement reduction on flow fluctuations. Results for maximum and minimum flow condition are reported in Figure 19. These results are also compared with the pressure ripple measurement of a commercial EGM design of a similar displacement taken as reference (blue curve, in both figures). Two important considerations can be derived from the plots 
of Figure 19: firstly, the flow oscillations of the VD-EGM are in line (even lower as compared to the reference, thanks to the asymmetric design of the teeth) with those realized by the commercial reference unit; secondly, it can be noticed that the pressure ripple at min displacement is higher than that at max displacement. This is in accordance to what was already predicted analytically by eq. (63).

\section{Conclusion}

This paper has presented an analytical study to describe the functioning of an innovative concept for economical variable delivery flow external gear machine (VD-EGM). The proposed principle of operation differs from those previously introduced for VD-EGM, which are based on moving the gears axially or radially, and it is based on the realization of a variable timing for the connections of the displacement chambers (the tooth space volumes, TSVs) with the inlet and outlet. While variable timing concepts for variable displacement machines were already successfully introduced for piston units, the implementation on the external gear machine architecture represent an element of novelty. In particular, the proposed variable timing concept is realized through the introduction of a movable element (slider) in lateral bushings of the EGM. Since the position of the slider directly determines the actual flow delivered by the unit, there is no need of fast acting elements (such as on/off valves) such as in other variable timing concepts for piston units.

While the concept was previously introduced by the authors and described through numerical simulations, in this paper an original analytical description is provided to describe the theoretical operation of the proposed VD-EGM. An energy method previously published for the case of standard EGMs with symmetric teeth was derived to provide general expressions for delivered flow rate, input shaft torque and the geometric displacement for the case of asymmetric gears. These expressions were then utilized in accordance to the principle of variation of the flow rate to describe the features of the VD-EGM.

The results of the analytical model show a very good agreement with the numerical analysis performed by the numerical simulation, showing the validity of the approach. In particular, the expected range of flow variation (as well as torque variation) of a gears previously found from a numerical optimization process were confirmed by the analytical model, showing a reduction in displacement from $100 \%$ to $68 \%$ of the maximum flow for the considered design. Both the numerical model and the analytical model show a torque reduction related to the flow reduction, highlighting the inherent efficiency of the proposed concept.

An interesting result of the analytical study is the description of the effect of the flow regulation on the instantaneous flow and torque pulsation, an aspect that was not particularly clear in the previous study on the VD-EGM performed by the authors. In particular, the analytical study permitted to describe the origin of an augmented tendency of flow and torque oscillations in condition of partial flow. This aspect was confirmed also by the numerical model.

All the considerations made above were also proven on the basis of a proof of concept test on the VD-EGM, for which an experimental campaign was performed to confirm both steady state and transient operating features of the proposed concept.

The present paper further supports the authors' research towards the formulation of a viable and economical concept for implementing cost effective and efficient VD-EGMs capable of operating in a high range of pressure and flow conditions.

\section{Acknowledgments}

This research was supported by the Center for Compact and Efficient Fluid Power (CCEFP), a National Science Foundation (NSF) Engineering Research Center (Project 1F.1) funded under cooperative agreement number EEC-0540834, and also by the NSF PFI:AIR - TT program (award n. 1543078).

\section{References}

[1] H. Murrenhoff, S. Sgro, M. Vukovic, 2014, An Overview of Energy Saving Architectures for Mobile Applications, 9th International Fluid Power Conference, March 24-26 2014, Aachen, Germany

[2]. M. Ivantysynova, 1998, Pump Controlled Actuator - a Realistic Alternative for Heavy Duty Manipulators and Robots, International Scientific Forum in Fluid Power Control of Machinery and Manipulators, Cracow, Fluid Power Net Publication, 1998, chapter 5, pp. $101-123$.

[3]. R.S. Devendran, A. Vacca, 2014, A novel design concept for variable delivery flow external gear pumps and motors. International Journal of Fluid Power, Sep 2, 2014, 15(3), 121-137. 
[4]. R.S. Devendran, A. Vacca, 2016, A Flow Control System for a Novel Concept of Variable Delivery External Gear Pump, 10th IFK International Fluid Power Conference, March 8-10, 2016, Dresden, Germany

[5]. T.E. Beacham, 1946, High Pressure Gear Pumps, Proc. I. Mech. E., 154.

[6]. C. Bonacini, 1961, Sulla Portata delle Pompe ad Ingranaggi, L'Ingegnere, Anno, Milan, Italy (in Italian).

[7]. J. Ivantysyn, M. Ivantysynova, 2003, Hydrostatic Pumps and Motors, Tech Books Int., New Delhi, India.

[8]. N.H. Manring, S.B. Kasaragadda, 2005, The theoretical flow ripple of an external gear pump, ASME J. Dynam Sys, Meas Control, 125.

[9]. S. Negrini, 1996, A gear pump designed for noise abatement and flow ripple reduction, Proceedings of the International Fluid Power Expositions and Technical Conference.

[10]. D. Schwuchow, 2002, Design tools for Modern External Gear Pumps, Proceedings of the 3rd International Fluid Power Conference, Aachen, Germany, Vol. 3, pp. 141-152.

[11]. M. Morselli, 2008, A Positive-displacement rotary pump with helical rotors, European Patent Application, EP 1132618.

[12]. M. Lätzel, D. Schwuchow, 2012, An innovative external gear pump for low noise applications, Proceedings of 8th International Fluid Power Conference, Dresden, Germany.

[13]. M. Morselli, 2005, Positive-Displacement Rotary Pump, Patent Publication Application, US 6887055.

[14]. M. Morselli, 2015, Geared hydraulic machine and relative gear wheel, Patent Application Publication, US 20150330387.

[15]. D. Yang, D. Zhong, 1987, Radial-Movable Variable Displacement Gear Pump (Motor), Chinese Patent Application, CN 85109203.

[16]. W. Reiners, G. Wiggermann, 1960, Variable Delivery Gear Pumps, The Patent Office London, GB968998.

[17]. L.F. Winmill, 2001, Adjustable-Displacement Gear Pump, Patent Application Publication, US2001024618.

[18]. E. Bussi, 1992, Variable Delivery Gear Pump, European Patent Application, EP0478514.

[19]. T. Hoji, S. Nagao, K. Shinozaki, 2008, Gear Pump, Patent Application Publication, US2008044308.

[20]. J.M. Clarke, 2002, Hydraulic Transformer using a Pair of Variable Displacement Gear Pumps, Patent Application Publication, US2002104313.

[21]. D. Voigt., 2011, Variable Flow Spur Gear Oil Pump for Utility Vehicles Engines, MTZ Worldwide eMagazine, Vol. 72, issue 4, pp.24-29

[22]. Ehsan M., Rampen, W.H.S., Salter S.H., 1997, Modeling of Digital-Displacement Pump-Motors and Their Application as Hydraulic Drives for Nonuniform Loads, J. Dyn. Sys., Meas., Control 122(1), pp 210-215

[23]. Mahrenholz J., Lumkes J.J., 2009, Model Development and Experimental Analysis of a Virtually Variable Displacement Pump System, International Journal of Fluid Power

[24]. R.S. Devendran, A. Vacca., 2015, A Novel Concept for a Variable Delivery External Gear Machine, 14th Scandinavian International Conference on Fluid Power, May 20-22, 2015, Tampere, Finland.

[25]. K. Nagamura, K. Ikejo, F.G. Tutulan, 2004, Design and performance of gear pumps with a non-involute tooth profile, Proceedings of the Institution of Mechanical Engineers, Part B: Journal of Engineering Manufacture, Vol. 218, pp. $699-711$.

[26]. F.L. Litvin, A. Fuentes, 2004, Gear Geometry and Applied Theory, 2nd edition, Cambridge University Press.

[27]. A. Vacca, M. Guidetti, 2011, Modelling and experimental validation of external spur gear machines for fluid power applications, Elsevier Simulation and Modelling Practice and Theory, vol. 19, pp. 2007-2031.

[28]. P. Casoli, A. Vacca and G. Berta, 2007, A Numerical Model for the Simulation of Flow in Hydraulic External Gear Machines, Power Transmission and Motion Control, Bath, UK.

[29]. A. Vacca, G. Franzoni, P. Casoli, 2007, On the Analysis of Experimental Data for External Gear Machines and their Comparison with Simulation Results, ASME International Mechanical Engineering Congress and Exposition, Seattle, (WA), USA. 